\title{
Decision-theoretic rough sets-based three-way approximations of interval-valued fuzzy sets
}

\author{
Guangming Lang ${ }^{a} *$ \\ ${ }^{a}$ School of Mathematics and Computer Science, Changsha University of Science and Technology \\ Changsha, Hunan 410082, P.R. China \\ ${ }^{b}$ College of Mathematics and Econometrics, Hunan University \\ Changsha, Hunan 410082, P.R. China
}

\begin{abstract}
In practical situations, interval-valued fuzzy sets are frequently encountered. In this paper, firstly, we present shadowed sets for interpreting and understanding interval fuzzy sets. We also provide an analytic solution to computing the pair of thresholds by searching for a balance of uncertainty in the framework of shadowed sets. Secondly, we construct errorsbased three-way approximations of interval-valued fuzzy sets. We also provide an alternative decision-theoretic formulation for calculating the pair of thresholds by transforming intervalvalued loss functions into single-valued loss functions, in which the required thresholds are computed by minimizing decision costs. Thirdly, we compute errors-based three-way approximations of interval-valued fuzzy sets by using interval-valued loss functions. Finally, we employ several examples to illustrate that how to take an action for an object with intervalvalued membership grade by using interval-valued loss functions.
\end{abstract}

Keywords: Decision-theoretic rough sets; Interval-valued fuzzy sets; Interval-valued loss function; Shadowed set

\section{Introduction}

Interval-valued fuzzy sets [31], as an extension of fuzzy sets [39], is a powerful mathematical tool to describe uncertainty information, in which the concept of the membership function using the subintervals of the interval $[0,1]$ as the set of membership grades is a fundamental notion. It has been intensively investigated, not only its theoretical aspects, but also its numerous applications, and the approximations of interval-valued fuzzy sets by using several levels of membership grades have became an important research direction.

Recently, researchers $[1,5,7,9,10,12,14-16,20-22,40]$ have investigated fuzzy sets from different aspects. For example, Pedrycz [22] proposed shadowed sets for interpreting fuzzy sets by using several

\footnotetext{
${ }^{*}$ Corresponding author. Tel./fax: +867318822855 ,

E-mail address: langguangming1984@126.com(G.M.Lang).
} 
levels of membership grades, in which, if the membership grade of an element is close to 1, it would be considered to be the same as 1 and elevated to 1 ; If the membership grade is close to 0 , it would be considered to be the same as 0 and is reduced to 0 ; If the membership grade is neither close to 0 nor close to 1 , it would be put into a shadowed region, in which the elevation and reduction operations use thresholds that provide semantically meaningful and acceptance levels of degree of closeness of membership values to 1 and 0 , respectively. Sequently, a lot of investigations [2-4, 8, 11, 19, 23-27,32,41] have been done on shadowed sets. For instance, Deng et al. [7] computed a pair of thresholds, whose interpretation and determination is a fundamental issue for expressing fuzzy sets, for three-way approximations of fuzzy sets by using loss functions, and classify a set of objects into three regions by using the pair of thresholds. In practical situations, interval-valued fuzzy sets whose membership functions are using the subintervals of the interval $[0,1]$ are of interest because such type of sets are frequently encountered. So far we have not seen the similar investigation on interval-valued fuzzy sets. Therefore, it is of interest to investigate that how to express interval-valued fuzzy sets as fuzzy sets.

To computing and interpreting a pair of thresholds, a lot of investigations [13-18,33-37] have been done on three-way decision-theory by using loss functions in literatures. For example, Li et al. [13] evaluated the cost and benefit of assigning an instance to a specific subcategory and defined a general loss function for supervised leaning. Liang et al. $[14,15]$ presented triangular fuzzy decision-theoretic rough sets and systematic studies on three-way decisions with interval-valued decision-theoretic rough sets. Liu et al. [17] proposed stochastic decision-theoretic rough sets, interval-valued decision-theoretic rough sets, fuzzy decision-theoretic rough sets and dynamic decision-theoretic rough sets. In practical situations, interval-valued loss functions as interval-valued numbers [16,28,29] are of interest because such functions are frequently encountered. Although interval-valued loss functions are complex in practice, we have not seen enough investigations on interval-valued fuzzy sets by using interval-valued loss functions so far. Therefore, it is urgent to further study interval-valued loss functions for making a decision by using threeway decision-theory.

The purpose of this paper is to further investigate interval-valued fuzzy sets. Section 2 introduces the basic principles of decision-theoretic rough sets, shadowed sets and decision-theoretic three-way approximations of fuzzy sets. Section 3 presents shadowed sets of interval-valued fuzzy sets. Section 4 is devoting to errors-based interpretation of shadowed sets of interval-valued fuzzy sets. Section 5 presents decisiontheoretic rough sets-based three-way approximations of interval-valued fuzzy sets by using transforming interval-valued loss functions into single loss functions. Section 6 investigates decision-theoretic rough sets-based three-way approximations of interval-valued fuzzy sets by using interval-valued loss functions from another view. The conclusion comes in Section 7. 


\section{Preliminaries}

In this section, we review some concepts of fuzzy sets, interval-valued fuzzy sets, shadowed sets and decision-theoretic three-way approximations of fuzzy sets.

\subsection{Shadowed sets of fuzzy sets}

In [39], Zadeh presented the concept of fuzzy sets for interpreting uncertainty problems.

Definition 2.1 [39] Let $\mu_{A}$ be a mapping from $U$ to $[0,1]$ such as $\mu_{A}: U \longrightarrow[0,1]: x \rightarrow \mu_{A}(x)$, where $x \in U, \mu_{A}$ is the membership function. Then $A$ is referred to as a fuzzy set.

In [22], Pedrycz presented the concept of shadowed sets for expressing fuzzy sets.

Definition 2.2 [22] Let A be a fuzzy set, the shadowed set $S_{\mu_{A}}$ of $A$ is defined as

$$
S_{\mu_{A}}(x)=\left\{\begin{array}{cc}
1, & \mu(x) \geq \alpha \\
0, & \mu(x) \leq \beta \\
{[0,1],} & \beta<\mu(x)<\alpha .
\end{array}\right.
$$

In Pedrycz's model, an optimal pair of thresholds is computed by minimizing the absolute difference as

$$
\begin{aligned}
V_{(\alpha, \beta)}\left(\mu_{A}\right) & =\mid \text { Elevated Area }(\alpha, \beta) \\
& =\left|\sum_{\mu_{A}(x) \geq \alpha}\left(1-\mu_{A}(x)\right)+\sum_{\mu_{A}(x) \leq \beta}\left(\mu_{A}(x)\right)-\operatorname{Card}\left(\left\{x \in U \mid \beta<\mu_{A}(x)<\alpha\right\}\right)\right|,
\end{aligned}
$$

where $\operatorname{card}(\cdot)$ denotes the cardinality of a set $\cdot$, and an optimal pair of thresholds $\alpha$ and $\beta$ can be derived by minimizing the objective function $V_{(\alpha, \beta)}\left(\mu_{A}\right)$. Similarly, it is also difficult to compute the pair of thresholds $\alpha$ and $\beta$ since minimizing $V_{(\alpha, \beta)}\left(\mu_{A}\right)$ involves two parameters $\alpha$ and $\beta$. For convenience, by using $\alpha+\beta=1$, the objective function is simplified into

$$
\begin{aligned}
V_{(\alpha, 1-\alpha)}\left(\mu_{A}\right) & =\mid \text { Elevated } \operatorname{Area}_{(\alpha, 1-\alpha)}\left(\mu_{A}\right)+\text { Reduced } \operatorname{Area}_{(\alpha, 1-\alpha)}\left(\mu_{A}\right)-\text { Shadowed } \operatorname{Area}_{(\alpha, 1-\alpha)}\left(\mu_{A}\right) \mid \\
& =\mid \sum_{\mu_{A}(x) \geq \alpha}\left(1-\mu_{A}(x)\right)+\sum_{\mu_{A}(x) \leq 1-\alpha}\left(\mu_{A}(x)\right)-\operatorname{Card}\left(\left\{x \in U \mid 1-\alpha\left(<\mu_{A}(x)<\alpha\right\}\right) \mid .\right.
\end{aligned}
$$

\subsection{Decision-theoretic three-way approximations of fuzzy sets}

In terms of the errors, Deng et al. [7] expressed the objective function to further investigate shadowed sets of fuzzy sets as

$$
\begin{aligned}
V_{(\alpha, \beta)}\left(\mu_{A}\right) & =\left|E_{e}\left(\mu_{A}\right)+E_{r}\left(\mu_{A}\right)-E_{s}\left(\mu_{A}\right)\right| \\
& =\left|\sum_{\mu_{A}(x) \geq \alpha}\left(1-\mu_{A}(x)\right)+\sum_{\mu_{A}(x) \leq \beta}\left(\mu_{A}(x)\right)-\sum_{\beta<\mu_{A}(x)<\alpha}\left(1-\mu_{A}(x)\right)+\sum_{\beta<\mu_{A}(x)<\alpha}\left(\mu_{A}(x)\right)\right| .
\end{aligned}
$$


The objective function is constructed on elevated area, reduced area and shadowed area, and it is necessary to investigate that which numeric value is meaningful to the membership grade of elements in the shadowed area.

By replacing the unit interval $[0,1]$ with 0.5 , Deng et al. provided

$$
T_{\mu_{A}}(x)=\left\{\begin{array}{cc}
1, & \mu(x) \geq \alpha \\
0, & \mu(x) \leq \beta \\
0.5, & \beta<\mu(x)<\alpha .
\end{array}\right.
$$

Subsequently, by analyzing $T_{\mu_{A}}(x)$, we have

$$
\begin{aligned}
E_{(\alpha, \beta)}\left(\mu_{A}\right) & =E_{e}\left(\mu_{A}\right)+E_{r}\left(\mu_{A}\right)+E_{S_{0.5}}\left(\mu_{A}\right) \\
& =\sum_{\mu_{A}(x) \geq \alpha}\left(1-\mu_{A}(x)\right)+\sum_{\mu_{A}(x) \leq \beta}\left(\mu_{A}(x)\right)-\sum_{0.5<\mu_{A}(x)<\alpha(t)}\left(\mu_{A}(x)-0.5\right)+\sum_{\beta<\mu_{A}(x)<0.5}\left(0.5-\mu_{A}(x)\right) .
\end{aligned}
$$

Correspondingly, the total error as the summation of errors of all objects are expressed as

$$
E_{(\alpha, \beta)}\left(\mu_{A}\right)=\sum_{x \in U} E_{(\alpha, \beta)}\left(\mu_{A}(x)\right)
$$

where

$$
E_{(\alpha, \beta)}\left(\mu_{A}(x)\right)=\left\{\begin{array}{cc}
1-\mu(x), & \mu(x) \geq \alpha \\
0.5-\mu(x), & \beta<\mu(x) \leq 0.5 \\
\mu(x)-0.5, & 0.5<\mu(x)<\alpha \\
\mu(x)-0, & \mu(x) \leq \beta
\end{array}\right.
$$

The total error is minimized by minimizing the error of each individual object, and we search for a pair of thresholds $\alpha$ and $\beta$ such that $E_{(\alpha, \beta)}\left(\mu_{A}(x)\right)$ is minimized for each object. We consider the following actions and associated errors for minimizing the error of each object:

(1) : elevate to $1: 1-\mu_{A}(x) ;(2)$ : reduce to $0: \mu_{A}(x)-0 ;(3):$ reduce or elevate to $0.5:\left|\mu_{A}(x)-0.5\right|$.

That is, the absolute differences between $\mu_{A}(x)$ and three values $1,0.5$ and 0 , respectively, are the associated errors. A minimized difference is obtained if $\mu_{A}(x)$ is changed into a value that is closest to $\mu_{A}(x)$.

Table 1: Loss function.

\begin{tabular}{ccccc}
\hline Action & Fuzzy set membership grade & Three-way membership grade & Error & Loss \\
\hline$a_{e}$ & $\mu_{A}(x) \geq \alpha$ & 1 & $1-\mu_{A}(x)$ & $\lambda_{e}$ \\
$a_{r}$ & $\mu_{A}(x) \leq \beta$ & 0 & $\mu_{A}(x)$ & $\lambda_{r}$ \\
$a_{s_{\downarrow}}$ & $0.5 \leq \mu_{A}(x)<\alpha$ & 0.5 & $\mu_{A}(x)-0.5$ & $\lambda_{s_{\downarrow}}$ \\
$a_{s_{\uparrow}}$ & $\beta<\mu_{A}(x)<0.5$ & 0.5 & $0.5-\mu_{A}(x)$ & $\lambda_{s_{\uparrow}}$ \\
\hline
\end{tabular}

By considering various costs of the actions of elevation and reduction, Deng et al. presented an analytic solution of computing the pair of thresholds $\alpha$ and $\beta$ by using loss functions. In Table 2, the set 
of actions $\left\{a_{e}, a_{r}, a_{s_{\downarrow}}, a_{s_{\uparrow}}\right\}$ describes four possible actions on changing the membership grade. The fuzzy membership grade $\mu_{A}(x)$ represents the state of object in the second column, and the errors of different actions are given in the fourth column, and the losses of different actions are given in the fifth column.

Suppose $\lambda_{e}>0, \lambda_{r}>0, \lambda_{s_{\downarrow}}>0, \lambda_{s_{\uparrow}}>0, \lambda_{s_{\downarrow}} \leq \lambda_{r}$ and $\lambda_{s_{\uparrow}} \leq \lambda_{e}$, we immediately have three rules as (E) If $\mu_{A}(x) \geq \alpha$, then $T_{\mu_{A}}(x)=1$; (R) If $\mu_{A}(x) \leq \beta$, then $T_{\mu_{A}}(x)=0$; (S) If $\beta<\mu_{A}(x)<\alpha$, then $T_{\mu_{A}}(x)=0.5$, where

$$
\alpha=\frac{2 \lambda_{e}+\lambda_{s_{\downarrow}}}{2\left(\lambda_{e}+\lambda_{s_{\downarrow}}\right)} \text { and } \beta=\frac{\lambda_{s_{\uparrow}}}{2\left(\lambda_{r}+\lambda_{s_{\uparrow}}\right)} \text {. }
$$

\section{Shadowed sets of interval-valued fuzzy sets and its errors-based inter- pretations}

In this section, we present the concept of shadowed sets of interval-valued fuzzy sets and its errorsbased interpretations for illustrating interval-valued fuzzy sets.

\subsection{Shadowed sets of interval-valued fuzzy sets}

In this subsection, we present the concept of shadowed sets of interval-valued fuzzy sets.

Definition 3.1 Let $D_{[0,1]}$ be the set of closed subintervals of the interval $[0,1]$. An interval-valued fuzzy set $A$ in $U$ is given by $A=\left\{\left(x, \widetilde{\mu}_{A}(x)\right) \mid x \in U\right\}$, where $\widetilde{\mu}_{A}: X \longrightarrow D_{[0,1]}: x \longrightarrow \widetilde{\mu}_{A}(x)=\left[\mu_{A}^{-}(x), \mu_{A}^{+}(x)\right]$.

Definition 3.2 Let $A$ be an interval-valued fuzzy set, $\widetilde{\mu}_{A}(x)=\left[\mu_{A}^{-}(x), \mu_{A}^{+}(x)\right]$ be a membership grade of $x \in U$, and $\theta \in[0,1]$. Then the transformed formula of $\widetilde{\mu}_{A}(x)$ is $\left.m_{\theta} \widetilde{\mu}_{A}(x)\right)=(1-\theta) \cdot \mu_{A}^{-}(x)+\theta \cdot \mu_{A}^{+}(x)$; Furthermore, $\left.A_{\theta}=\left\{\left(x, m_{\theta} \widetilde{\mu}_{A}(x)\right)\right) \mid x \in U\right\}$.

Example 3.3 (1) Let $\widetilde{\mu}_{A}(x)=[0.1,0.2]$ and $\widetilde{\mu}_{B}(x)=[0.15,0.25]$ for $x \in U$, and $\theta=0.5$. Then

$$
\begin{aligned}
& \left.m_{\theta} \widetilde{\mu}_{A}(x)\right)=(1-0.5) \times 0.1+0.5 \times 0.2=0.15 \\
& \left.m_{\theta} \widetilde{\mu}_{B}(x)\right)=(1-0.5) \times 0.15+0.5 \times 0.25=0.2 .
\end{aligned}
$$

(2) Let $A=\frac{x_{1}}{\bar{\mu}_{A}\left(x_{1}\right)}+\frac{x_{2}}{\tilde{\mu}_{A}\left(x_{2}\right)}+\frac{x_{3}}{\bar{\mu}_{A}\left(x_{3}\right)}+\frac{x_{4}}{\bar{\mu}_{A}\left(x_{4}\right)}$ be an interval-valued fuzzy set, where $\widetilde{\mu}_{A}\left(x_{1}\right)=[0.1,0.2], \widetilde{\mu}_{A}\left(x_{2}\right)=$ $[0.6,0.8], \widetilde{\mu}_{A}\left(x_{3}\right)=[0.3,0.5]$ and $\widetilde{\mu}_{A}\left(x_{4}\right)=[0.8,0.1]$. If we take $\theta=0.5$, then $A_{\theta}=\frac{x_{1}}{0.15}+\frac{x_{2}}{0.7}+\frac{x_{3}}{0.4}+\frac{x_{4}}{0.45}$.

For simplicity, we denote $\widetilde{\mu}_{A}$ as $\mu_{A}$ in the following discussion.

Definition 3.4 Let A be an interval-valued fuzzy set, then the shadowed set $S_{\mu_{A}}$ of A is defined as

$$
S_{\mu_{A}}(x)=\left\{\begin{array}{cc}
1, & m_{\theta}\left(\mu_{A}(x)\right) \geq \alpha \\
0, & m_{\theta}\left(\mu_{A}(x) \leq \beta\right. \\
{[0,1],} & \beta<m_{\theta}\left(\mu_{A}(x)\right)<\alpha
\end{array}\right.
$$


For an object $x$, we elevate the membership grade from $\mu_{A}(x)$ to 1 if $m_{\theta}\left(\mu_{A}(x)\right) \geq \alpha$; We reduce the membership grade from $\mu_{A}(x)$ to 0 if $m_{\theta}\left(\mu_{A}(x)\right) \leq \beta$; We change the membership grade from $\mu_{A}(x)$ to $[0,1]$ if $\beta<m_{\theta}\left(\mu_{A}(x)\right)<\alpha$.

The pair of thresholds $\alpha$ and $\beta$ are important for computing three-way approximations of intervalvalued fuzzy sets. In what follows, we introduce a systematic way to compute the pair of thresholds $\alpha$ and $\beta$ by minimizing an objective function as

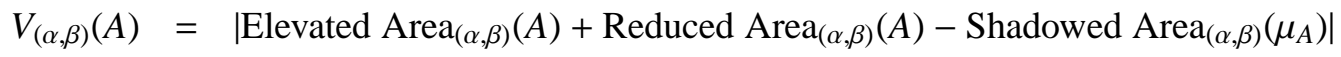

$$
\begin{aligned}
& =\left|\sum_{m_{\theta}\left(\mu_{A}(x)\right) \geq \alpha}\left(1-m_{\theta}\left(\mu_{A}(x)\right)\right)+\sum_{m_{\theta}\left(\mu_{A}(x)\right) \leq \beta}\left(m_{\theta}\left(\mu_{A}(x)\right)\right)-\operatorname{Card}\left(\left\{x \in U \mid \beta<m_{\theta}\left(\mu_{A}(x)\right)<\alpha\right\}\right)\right|,
\end{aligned}
$$

where $\operatorname{card}(\cdot)$ denotes the cardinality of a set $\cdot$, and an optimal pair of thresholds $\alpha$ and $\beta$ can be derived by minimizing the objective function $V_{(\alpha, \beta)}(A)$. Similarly, minimizing $V_{(\alpha, \beta)}(A)$ involves two parameters $\alpha$ and $\beta$. For convenience, by assuming that $\alpha+\beta=1$, the objective function is simplified into

$$
\begin{aligned}
& V_{(\alpha, 1-\alpha)}(A) \\
& =\mid \text { Elevated Area }_{(\alpha, 1-\alpha)}\left(m_{\theta}\left(\mu_{A}(x)\right)\right)+\text { Reduced } \operatorname{Area}_{(\alpha, 1-\alpha)}\left(m_{\theta}\left(\mu_{A}(x)\right)\right)-\text { Shadowed Area }_{(\alpha, 1-\alpha)}\left(m_{\theta}\left(\mu_{A}(x)\right)\right) \mid \\
& =\left|\sum_{m_{\theta}\left(\mu_{A}(x)\right)(x) \geq \alpha}\left(1-m_{\theta}\left(\mu_{A}(x)\right)\right)+\sum_{m_{\theta}\left(\mu_{A}(x)\right) \leq 1-\alpha}\left(m_{\theta}\left(\mu_{A}(x)\right)\right)-\operatorname{Card}\left(\left\{x \in U \mid 1-\alpha<m_{\theta}\left(\mu_{A}(x)\right)<\alpha\right\}\right)\right| .
\end{aligned}
$$

There exist two interpretations of shadowed sets of interval-valued fuzzy sets. In a wide sense, a shadowed set is a three-valued fuzzy set, which is used to approximate an interval-valued fuzzy set. In a narrow sense, we interpret the notion of a shadowed set according to its exact formulation, namely, the choice of the set of membership grades $\{0,[0,1], 1\}$ and the objective function. Therefore, shadowed sets of interval-valued fuzzy sets are examples of three-way approximations of interval-valued fuzzy sets.

\subsection{Errors-based interpretation of shadowed sets for interval-valued fuzzy sets}

In this section, we present a detailed analysis of a objective function for shadowed sets of intervalvalued fuzzy sets in terms of errors of approximations. We also provide a new objective function by the total error of approximations for determining the thresholds $\alpha$ and $\beta$.

To further study shadowed sets of interval-valued fuzzy sets, we express the objective function in terms of the errors introduced by a shadowed set approximation. For an object $x$ with membership grade $m_{\theta}\left(\mu_{A}(x)\right)$, the elevation operation changes the membership grade from $m_{\theta}\left(\mu_{A}(x)\right)$ to 1 , the reduction operation changes the membership grade from $m_{\theta}\left(\mu_{A}(x)\right)$ to 0 , and the errors induced by elevation and reduction are shown as

$$
E_{e}\left(\mu_{A}(x)\right)=1-m_{\theta}\left(\mu_{A}(x)\right), E_{r}\left(\mu_{A}(x)\right)=m_{\theta}\left(\mu_{A}(x)\right)
$$

The errors $E_{e}\left(\mu_{A}\right)$ and $E_{r}\left(\mu_{A}\right)$ induced by the elevation and reduction operations for an interval-valued 
fuzzy set $A$ of the universe $U$, respectively, are shown as

$$
E_{e}\left(\mu_{A}\right)=\sum_{m_{\theta}\left(\mu_{A}(x)\right) \geq \alpha}\left(1-m_{\theta}\left(\mu_{A}(x)\right)\right), E_{r}\left(\mu_{A}(x)\right)=\sum_{m_{\theta}\left(\mu_{A}(x)\right) \leq \beta} m_{\theta}\left(\mu_{A}(x)\right) .
$$

The error for the shadowed area is not clear because of the unit interval $[0,1]$ as the membership grade when $\beta<m_{\theta}\left(\mu_{A}(x)\right)<\alpha$. By computing the difference between $m_{\theta}\left(\mu_{A}(x)\right)$ and the maximum 1 and the minimum value 0 and summarizing them up, we have

$$
E_{s}\left(\mu_{A}\right)=\sum_{\beta<m_{\theta}\left(\mu_{A}(x)\right)<\alpha}\left(1-m_{\theta}\left(\mu_{A}(x)\right)\right)+\sum_{\beta<m_{\theta}\left(\mu_{A}(x)\right)<\alpha} m_{\theta}\left(\mu_{A}(x)\right) .
$$

Subsequently, we express the objective function in terms of errors by using the error-based interpretation of the three areas as

$$
\begin{aligned}
& V_{(\alpha, \beta)}\left(\mu_{A}\right) \\
& =\left|E_{e}\left(\mu_{A}\right)+E_{r}\left(\mu_{A}\right)-E_{s}\left(\mu_{A}\right)\right| \\
& =\mid \sum_{m_{\theta}\left(\mu_{A}(x)\right) \geq \alpha}\left(1-m_{\theta}\left(\mu_{A}(x)\right)\right)+\sum_{m_{\theta}\left(\mu_{A}(x)\right) \leq \beta} m_{\theta}\left(\mu_{A}(x)-\sum_{\beta<m_{\theta}\left(\mu_{A}(x)\right)<\alpha}\left(1-m_{\theta}\left(\mu_{A}(x)\right)\right)+\sum_{\beta<m_{\theta}\left(\mu_{A}(x)\right)<\alpha}\left(m_{\theta} \mu_{A}(x)\right) \mid .\right.
\end{aligned}
$$

The objective function is a kind of trade-off of errors produced by three regions. But the rationale for such a trade-off is not entirely clear. On one hand, $E_{s}\left(m_{\theta}\left(\mu_{A}(x)\right)\right)$ consists the errors of elevation and reduction operations, and it is impossible to elevate $m_{\theta}\left(\mu_{A}(x)\right)$ to 1 and reduce $m_{\theta}\left(\mu_{A}(x)\right)$ to 0 if $\beta<$ $m_{\theta}\left(\mu_{A}(x)\right)<\alpha$ simultaneously. On the other hand, we are not able to allocate any numeric membership grade for the elements in the shadowed area. In other words, any numeric value of the unit interval $[0,1]$ could be permitted to reflect the uncertainty. Therefore, it is necessary to investigate that which numeric value is meaningful to the membership grade of elements in the shadowed area.

Below, we present a three-way approximation of an interval-valued fuzzy set by replacing the unit interval $[0,1]$ with 0.5 as,

$$
T_{\mu_{A}}(x)=\left\{\begin{array}{cc}
1, & m_{\theta}\left(\mu_{A}(x)\right) \geq \alpha \\
0, & m_{\theta}\left(\mu_{A}(x)\right) \leq \beta \\
0.5, & \beta<m_{\theta}\left(\mu_{A}(x)\right)<\alpha
\end{array}\right.
$$

By analyzing $T_{\mu_{A}}(x)$, we see that the correspondences between areas of elevation and reduction and errors of elevation and reduction remain to be the same. But we need to revise the errors of the shadowed region as

$$
E_{S_{0.5}}\left(\mu_{A}\right)=\sum_{0.5<m_{\theta}\left(\mu_{A}(x)\right)<\alpha}\left(1-m_{\theta}\left(\mu_{A}(x)\right)\right)+\sum_{\beta<m_{\theta}\left(\mu_{A}(x)\right)<0.5}\left(m_{\theta}\left(\mu_{A}(x)\right)\right)
$$


By using $E_{e}\left(\mu_{A}\right), E_{r}\left(\mu_{A}\right)$ and $E_{s_{0.5}}\left(\mu_{A}\right)$, we have

$$
\begin{aligned}
E_{(\alpha, \beta)}\left(\mu_{A}\right)= & E_{e}\left(\mu_{A}\right)+E_{r}\left(\mu_{A}\right)+E_{S_{0.5}}\left(\mu_{A}\right) \\
= & \sum_{m_{\theta}\left(\mu_{A}(x)\right) \geq \alpha}\left(1-m_{\theta}\left(\mu_{A}(x)\right)+\sum_{m_{\theta}\left(\mu_{A}(x)\right) \leq \beta}\left(m_{\theta}\left(\mu_{A}(x)\right)\right)-\sum_{0.5<m_{\theta}\left(\mu_{A}(x)\right)<\alpha(t)}\left(m_{\theta}\left(\mu_{A}(x)\right)-0.5\right)\right. \\
& +\sum_{\beta<m_{\theta}\left(\mu_{A}(x)\right)<0.5}\left(0.5-m_{\theta}\left(\mu_{A}(x)\right)\right) .
\end{aligned}
$$

The total errors of the three areas are minimized instead of searching for a trade-off between different areas. Correspondingly, we express the total error as the summation of errors of all objects as

$$
E_{(\alpha, \beta)}\left(\mu_{A}\right)=\sum_{x \in U} E_{(\alpha, \beta)}\left(m_{\theta}\left(\mu_{A}(x)\right)\right)
$$

where

$$
E_{(\alpha, \beta)}\left(\mu_{A}(x)\right)=\left\{\begin{array}{cc}
1-m_{\theta}\left(\mu_{A}(x)\right), & m_{\theta}\left(\mu_{A}(x)\right) \geq \alpha \\
0.5-m_{\theta}\left(\mu_{A}(x)\right), & \beta<m_{\theta}\left(\mu_{A}(x)\right) \leq 0.5 \\
m_{\theta}\left(\mu_{A}(x)\right)-0.5, & 0.5<m_{\theta}\left(\mu_{A}(x)\right)<\alpha \\
m_{\theta}\left(\mu_{A}(x)\right), & m_{\theta}\left(\mu_{A}(x)\right) \leq \beta
\end{array}\right.
$$

The total error will be minimized by minimizing the error of each individual object, and we can search for a pair of thresholds $\alpha$ and $\beta$ such that $E_{(\alpha, \beta)}\left(\mu_{A}(x)\right)$ is minimized for each object. We consider the following actions and associated errors for minimizing the error of each object:

(1) : elevate to $1: 1-m_{\theta}\left(\mu_{A}(x)\right) ;(2):$ reduce to $0: m_{\theta}\left(\mu_{A}(x)\right)-0 ;(3):$ reduce or elevate to $0.5:\left|m_{\theta}\left(\mu_{A}(x)\right)-0.5\right|$.

That is, the absolute differences between $m_{\theta}\left(\mu_{A}(x)\right)$ and three values $1,0.5$ and 0 , respectively, are the associated errors. A minimized difference is obtained if $m_{\theta}\left(\mu_{A}(x)\right)$ is changed into a value that is closest to $m_{\theta}\left(\mu_{A}(x)\right)$.

\section{Decision-theoretic rough sets-based three-way approximations of interval- valued fuzzy sets}

In this section, we introduce a framework for decision-theoretic rough sets-based three-way approximations of interval-valued fuzzy sets.

\subsection{Cost-sensitive three-way approximations of interval-valued fuzzy sets}

In Section 3, we investigate three-way approximation of interval-valued fuzzy sets by using three membership grades of $0,0.5$ and 1 . We take one of the following three actions for an object with a membership grade: elevate the membership grade to 1 , reduce the membership grade to 0 , and change the membership grade to 0.5 . More specially, there are two situations for the third case: reduce the 
Table 2: Loss function

\begin{tabular}{ccccc}
\hline Action & Membership grade & Three-way membership grade & Error & Loss \\
\hline$a_{e}$ & $m_{\theta}\left(\mu_{A}(x)\right) \geq \alpha$ & 1 & $1-m_{\theta}\left(\mu_{A}(x)\right)$ & $\lambda_{e}$ \\
$a_{r}$ & $m_{\theta}\left(\mu_{A}(x)\right) \leq \beta$ & 0 & $m_{\theta}\left(\mu_{A}(x)\right)$ & $\lambda_{r}$ \\
$a_{s_{\downarrow}}$ & $0.5 \leq m_{\theta}\left(\mu_{A}(x)\right)<\alpha$ & 0.5 & $m_{\theta}\left(\mu_{A}(x)\right)-0.5$ & $\lambda_{s_{\downarrow}}$ \\
$a_{s_{\uparrow}}$ & $\beta<m_{\theta}\left(\mu_{A}(x)\right)<0.5$ & 0.5 & $0.5-m_{\theta}\left(\mu_{A}(x)\right)$ & $\lambda_{s_{\uparrow}}$ \\
\hline
\end{tabular}

membership grade to 0.5 if $m_{\theta}\left(\mu_{A}(x)\right) \geq 0.5$ and elevate the membership grade to 0.5 if $m_{\theta}\left(\mu_{A}(x)\right)<0.5$. Each action will incur error and the costs of different actions are not necessarily the same.

Table 2 summarizes information about three-way approximations of an interval-valued fuzzy set. Concretely, the set of actions $\left\{a_{e}, a_{r}, a_{s_{\downarrow}}, a_{s_{\uparrow}}\right\}$ describes four possible actions on changing the membership grade. For simplicity, we also use $\left\{e, r, s_{\downarrow}, s_{\uparrow}\right\}$ to denote the four actions. The elevation action $a_{e}$ elevate the membership grade of $x$ from $m_{\theta}\left(\mu_{A}(x)\right)$ to 1 , the reduction action $a_{r}$ reduce the membership grade of $x$ from $m_{\theta}\left(\mu_{A}(x)\right)$ to 0 , the elevation $a_{s \uparrow}$ elevate the membership grade of $x$ from $m_{\theta}\left(\mu_{A}(x)\right)$ to 0.5 if $m_{\theta}\left(\mu_{A}(x)\right)<0.5$, the reduction $a_{s_{\downarrow}}$ reduce the membership grade of $x$ from $m_{\theta}\left(\mu_{A}(x)\right)$ to 0.5 if $m_{\theta}\left(\mu_{A}(x)\right)>0.5$. The fuzzy membership grade $m_{\theta}\left(\mu_{A}(x)\right)$ represents the state of object in the second column, and the errors of different actions are given in the fourth column, and the losses of different actions are given in the fifth column.

Each of the four losses $\lambda_{e}, \lambda_{r}, \lambda_{s_{\downarrow}}$ and $\lambda_{s_{\uparrow}}$ provides the unit cost, and the actual cost of each action is weighted by the magnitude of its error. Suppose $R_{a}(x)=\lambda_{a} E_{a}\left(\mu_{A}(x)\right)$ denote the loss for taking actions $\left\{e, r, s_{\downarrow}, s_{\uparrow}\right\}$, the losses of four actions for an object can be computed as

$$
\begin{aligned}
R_{e}(x) & =\lambda_{e} E_{e}\left(\mu_{A}(x)\right)=\left(1-m_{\theta}\left(\mu_{A}(x)\right)\right) \lambda_{e} ; \\
R_{r}(x) & =\lambda_{r} E_{r}\left(\mu_{A}(x)\right)=m_{\theta}\left(\mu_{A}(x)\right) \lambda_{r} ; \\
R_{s_{\downarrow}}(x) & =\lambda_{s_{\downarrow}} E_{s_{\downarrow}}\left(m_{\theta}\left(\mu_{A}(x)\right)\right)=\left(m_{\theta}\left(\mu_{A}(x)\right)-0.5\right) \lambda_{s_{\downarrow}} ; \\
R_{s_{\uparrow}}(x) & =\lambda_{s_{\uparrow}} E_{s_{\uparrow}}\left(m_{\theta}\left(\mu_{A}(x)\right)\right)=\left(0.5-m_{\theta}\left(\mu_{A}(x)\right)\right) \lambda_{s_{\uparrow}} .
\end{aligned}
$$

Since only an action is taken for each object, the total loss of the approximation is computed by

$$
R=\sum_{x \in U} R_{a}(x)=\sum_{x \in U} \lambda_{a} E_{a}\left(\mu_{A}(x)\right)
$$

To minimize the total loss $R$, we take an action $\tau(x)$ that minimizes the loss $R_{a}(x)$ for each object, and $\tau(x)$ is a solution to the following minimization problem as

$$
\arg \min _{a \in a c t i o n} R_{a}(x),
$$

where $a \in\left\{e, r, s_{\downarrow}, s_{\uparrow}\right\}$. 
According to the value $\mu_{A}(x)$ of an object $x$, we have two groups of decision rules for obtaining threeway approximations of an interval-valued fuzzy set as follows:

(1) When $m_{\theta}\left(\mu_{A}(x)\right) \geq 0.5$, (E1) If $R\left(a_{e} \mid x\right) \leq R\left(a_{r} \mid x\right)$ and $R\left(a_{e} \mid x\right) \leq R\left(a_{s_{\downarrow}} \mid x\right)$, then take action $a_{e}$; (R1) If $R\left(a_{r} \mid x\right) \leq R\left(a_{e} \mid x\right)$ and $R\left(a_{r} \mid x\right) \leq R\left(a_{s_{\downarrow}} \mid x\right)$, then take action $a_{r} ;(S 1)$ If $R\left(a_{s_{\downarrow}} \mid x\right) \leq R\left(a_{e} \mid x\right)$ and $R\left(a_{s_{\downarrow}} \mid x\right) \leq R\left(a_{r} \mid x\right)$, then take action $a_{s_{\downarrow}}$.

(2) When $m_{\theta}\left(\mu_{A}(x)\right)<0.5$, (E2) If $R\left(a_{e} \mid x\right) \leq R\left(a_{r} \mid x\right)$ and $R\left(a_{e} \mid x\right) \leq R\left(a_{s_{\uparrow}} \mid x\right)$, then take action $a_{e}$; (R2) If $R\left(a_{r} \mid x\right) \leq R\left(a_{e} \mid x\right)$ and $R\left(a_{r} \mid x\right) \leq R\left(a_{s_{\uparrow}} \mid x\right)$, then take action $a_{r} ;(S 2)$ : If $R\left(a_{s_{\uparrow}} \mid x\right) \leq R\left(a_{e} \mid x\right)$ and $R\left(a_{s_{\uparrow}} \mid x\right) \leq R\left(a_{r} \mid x\right)$, then take action $a_{s_{\uparrow}}$.

\subsection{Single-valued loss functions-based three-way approximations of interval-valued fuzzy sets}

In this subsection, we consider loss functions satisfying certain properties for obtaining an analytic solution defining a three-way approximation.

Suppose (c1): $\lambda_{e}>0, \lambda_{r}>0, \lambda_{s_{\downarrow}}>0, \lambda_{s_{\uparrow}}>0 ;(c 2): \lambda_{s_{\downarrow}} \leq \lambda_{r} ;(c 3): \lambda_{s_{\uparrow}} \leq \lambda_{e}$, Condition $(c 1)$ requires that all costs are nonnegative; Condition (c2) illustrates that reducing a membership grade $\mu_{A}(x) \geq 0.5$ to 0.5 represents a smaller adjustment than reducing it to 0 , and a smaller cost is associated with action $a_{s_{\downarrow}}$; Condition (c3) illustrates that elevating a membership grade $\mu_{A}(x)<0.5$ to 0.5 represents a smaller adjustment than elevating it to 1 , and a smaller cost is associated with action $a_{s_{\uparrow}}$. With the assumptions $(c 1)-(c 3)$, we simplify the decision rules as follows:

(1) When $m_{\theta}\left(\mu_{A}(x)\right) \geq 0.5$, the rule $(E 1)$ is expressed as

$$
\begin{aligned}
R\left(a_{e} \mid x\right) \leq R\left(a_{r} \mid x\right) & \Leftrightarrow \quad\left(1-m_{\theta}\left(\mu_{A}(x)\right)\right) \lambda_{e} \leq m_{\theta}\left(\mu_{A}(x)\right) \lambda_{r} \\
& \Leftrightarrow \mu_{A}(x) \geq \frac{\lambda_{e}}{\lambda_{e}+\lambda_{r}}=\gamma ; \\
R\left(a_{e} \mid x\right) \leq R\left(a_{s_{\downarrow}} \mid x\right) & \Leftrightarrow \quad\left(1-m_{\theta}\left(\mu_{A}(x)\right)\right) \lambda_{e} \leq\left(m_{\theta}\left(\mu_{A}(x)\right)-0.5\right) \lambda_{s_{\downarrow}} \\
& \Leftrightarrow \quad m_{\theta}\left(\mu_{A}(x)\right) \geq \frac{2 \lambda_{e}+\lambda_{s_{\downarrow}}}{2\left(\lambda_{e}+\lambda_{s_{\downarrow}}\right)}=\alpha .
\end{aligned}
$$

The rule $R(1)$ is expressed by

$$
\begin{aligned}
R\left(a_{r} \mid x\right) \leq R\left(a_{r} \mid x\right) & \Leftrightarrow m_{\theta}\left(\mu_{A}(x)\right) \leq \gamma ; \\
R\left(a_{r} \mid x\right) \leq R\left(a_{s_{\downarrow}} \mid x\right) & \Leftrightarrow \quad m_{\theta}\left(\mu_{A}(x)\right) \lambda_{r} \leq\left(m_{\theta}\left(\mu_{A}(x)\right)-0.5\right) \lambda_{s_{\downarrow}} \\
& \Leftrightarrow \quad m_{\theta}\left(\mu_{A}(x)\right) \leq \frac{-\lambda_{s_{\downarrow}}(t)}{2\left(\lambda_{r}-\lambda_{s_{\downarrow}}(t)\right)}=\gamma^{-} .
\end{aligned}
$$

The rule $S(1)$ is expressed by

$$
R\left(a_{s_{\downarrow}} \mid x\right) \leq R\left(a_{e} \mid x\right) \Leftrightarrow m_{\theta}\left(\mu_{A}(x)\right) \leq \alpha ; R\left(a_{s_{\downarrow}} \mid x\right) \leq R\left(a_{s_{\downarrow}} \mid x\right) \Leftrightarrow m_{\theta}\left(\mu_{A}(x)\right) \geq \gamma^{-} .
$$


Since $\gamma^{-} \leq 0$ contradicts with the assumption $m_{\theta}\left(\mu_{A}(x)\right) \geq 0.5$, it is impossible to apply rule (R1) for reducing membership values. Therefore, when $m_{\theta}\left(\mu_{A}(x)\right) \geq 0.5$, the rules are simply expressed as (E1) If $m_{\theta}\left(\mu_{A}(x)\right) \geq \alpha$, then $T_{\mu_{A}}(x)=1$; (S1) If $0.5 \leq m_{\theta}\left(\mu_{A}(x)\right)<\alpha$, then $T_{\mu_{A}}(x)=0.5$.

(2) When $m_{\theta}\left(\mu_{A}(x)\right)<0.5$, the rule $(E 2)$ is expressed as

$$
\begin{aligned}
R\left(a_{e} \mid x\right) \leq R\left(a_{r} \mid x\right) & \Leftrightarrow \quad\left(1-m_{\theta}\left(\mu_{A}(x)\right)\right) \lambda_{e} \leq m_{\theta}\left(\mu_{A}(x)\right) \lambda_{r} \\
& \Leftrightarrow m_{\theta}\left(\mu_{A}(x)\right) \geq \frac{\lambda_{e}}{\lambda_{e}+\lambda_{r}}=\gamma ; \\
R\left(a_{e} \mid x\right) \leq R\left(a_{s_{\uparrow}} \mid x\right) & \Leftrightarrow \quad\left(1-m_{\theta}\left(\mu_{A}(x)\right)\right) \lambda_{e} \leq\left(0.5-m_{\theta}\left(\mu_{A}(x)\right)\right) \lambda_{s \uparrow} \\
& \Leftrightarrow \quad m_{\theta}\left(\mu_{A}(x)\right) \geq \frac{\lambda_{e}-0.5 \lambda_{s_{\uparrow}}}{\lambda_{e}-\lambda_{s_{\uparrow}}}=\gamma^{+} .
\end{aligned}
$$

The rule $R(2)$ is expressed as

$$
\begin{aligned}
R\left(a_{r} \mid x\right) \leq R\left(a_{e} \mid x\right) & \Leftrightarrow m_{\theta}\left(\mu_{A}(x)\right) \leq \gamma ; \\
R\left(a_{r} \mid x\right) \leq R\left(a_{s_{\uparrow}} \mid x\right) & \Leftrightarrow \quad m_{\theta}\left(\mu_{A}(x)\right) \lambda_{r} \leq\left(0.5-m_{\theta}\left(\mu_{A}(x)\right)\right) \lambda_{s_{\uparrow}} \\
& \Leftrightarrow \quad m_{\theta}\left(\mu_{A}(x)\right) \leq \frac{\lambda_{s_{\uparrow}}}{2\left(\lambda_{r}+\lambda_{s_{\uparrow}}\right)}=\beta .
\end{aligned}
$$

The rule $S(2)$ is expressed as

$$
R\left(a_{s_{\uparrow}} \mid x\right) \leq R\left(a_{e} \mid x\right) \Leftrightarrow m_{\theta}\left(\mu_{A}(x)\right) \leq \gamma^{+} ; R\left(a_{s_{\uparrow}} \mid x\right) \leq R\left(a_{s_{\uparrow}} \mid x\right) \Leftrightarrow m_{\theta}\left(\mu_{A}(x)\right) \geq \beta .
$$

Since $\gamma^{+} \geq 1$ contradicts with the assumption $m_{\theta}\left(\mu_{A}(x)\right)<0.5$, it is impossible to apply rule (E2) for elevating membership values. Therefore, when $m_{\theta}\left(\mu_{A}(x)\right)<0.5$, the remaining rules are simply expressed as (R2) If $m_{\theta}\left(\mu_{A}(x)\right) \leq \beta$, then $T_{\mu_{A}}(x)=0$; (S2) If $\beta \leq m_{\theta}\left(\mu_{A}(x)\right)<0.5$, then $T_{\mu_{A}}(x)=0.5$.

By combining the two sets of rules, we immediately have three rules as (E) If $m_{\theta}\left(\mu_{A}(x)\right) \geq \alpha(t)$, then $T_{\mu_{A}}(x)=1$; (R) If $m_{\theta}\left(\mu_{A}(x)\right) \leq \beta$, then $T_{\mu_{A}}(x)=0$; (S) If $\beta<m_{\theta}\left(\mu_{A}(x)\right)<\alpha$, then $T_{\mu_{A}}(x)=0.5$, where

$$
\alpha=\frac{2 \lambda_{e}+\lambda_{s_{\downarrow}}}{2\left(\lambda_{e}(t)+\lambda_{s_{\downarrow}}\right)} \text { and } \beta=\frac{\lambda_{s_{\uparrow}}}{2\left(\lambda_{r}+\lambda_{s_{\uparrow}}\right)} .
$$

\section{Interval-valued loss functions-based three-way approximations of interval- valued fuzzy sets: I}

In this section, we introduce a framework for interval-valued loss functions-based three-way approximations of interval-valued fuzzy sets.

\subsection{Cost-sensitive three-way approximations of interval-valued fuzzy sets}

In Section 4, we investigate three-way approximation of interval-valued fuzzy sets by using three membership grades of $0,0.5$ and 1 . We take one of the following three actions for an object with a 
membership grade: elevate the membership grade to 1 , reduce the membership grade to 0 , and change the membership grade to 0.5. More specially, there are two situations for the third case: reduce the membership grade to 0.5 if $m_{\theta}\left(\mu_{A}(x)\right) \geq 0.5$ and elevate the membership grade to 0.5 if $m_{\theta}\left(\mu_{A}(x)\right)<0.5$. Each action will incur error and the costs of different actions are not necessarily the same.

Table 3: Interval-valued loss function.

\begin{tabular}{ccccc}
\hline Action & Membership grade & Three-way membership grade & Error & Loss \\
\hline$a_{e}$ & $m_{\theta}\left(\mu_{A}(x)\right) \geq \alpha$ & 1 & $1-m_{\theta}\left(\mu_{A}(x)\right)$ & $\widetilde{\lambda}_{e}=\left[\lambda_{e}^{-}, \lambda_{e}^{+}\right]$ \\
$a_{r}$ & $m_{\theta}\left(\mu_{A}(x)\right) \leq \beta$ & 0 & $m_{\theta}\left(\mu_{A}(x)\right)$ & $\widetilde{\lambda}_{r}=\left[\lambda_{r}^{-}, \lambda_{r}^{+}\right]$ \\
$a_{s_{\downarrow}}$ & $0.5 \leq m_{\theta}\left(\mu_{A}(x)\right)<\alpha$ & 0.5 & $m_{\theta}\left(\mu_{A}(x)\right)-0.5$ & $\widetilde{\lambda}_{s_{\downarrow}}=\left[\lambda_{s_{\downarrow}}^{-}, \lambda_{s_{\downarrow}}^{+}\right]$ \\
$a_{s_{\uparrow}}$ & $\beta<m_{\theta}\left(\mu_{A}(x)\right)<0.5$ & 0.5 & $0.5-m_{\theta}\left(\mu_{A}(x)\right)$ & $\widetilde{\lambda}_{s \uparrow}=\left[\lambda_{s \uparrow}^{-}, \lambda_{s \uparrow}^{+}\right]$ \\
\hline
\end{tabular}

Table 3 summarizes information about three-way approximations of an interval-valued fuzzy set. Concretely, the set of actions $\left\{a_{e}, a_{r}, a_{s_{\downarrow}}, a_{s \uparrow}\right\}$ describes four possible actions on changing the membership grade. For simplicity, we also use $\left\{e, r, s_{\downarrow}, s_{\uparrow}\right\}$ to denote the four actions. Concretely, the elevation action $a_{e}$ elevate the membership grade of $x$ from $m_{\theta}\left(\mu_{A}(x)\right)$ to 1 , the reduction action $a_{r}$ reduce the membership grade of $x$ from $m_{\theta}\left(\mu_{A}(x)\right)$ to 0 , the elevation $a_{s_{\uparrow}}$ elevate the membership grade of $x$ from $m_{\theta}\left(\mu_{A}(x)\right)$ to 0.5 if $m_{\theta}\left(\mu_{A}(x)\right)<0.5$, the reduction $a_{s_{\downarrow}}$ reduce the membership grade of $x$ from $m_{\theta}\left(\mu_{A}(x)\right)$ to 0.5 if $m_{\theta}\left(\mu_{A}(x)\right)>0.5$. The fuzzy membership grade $m_{\theta}\left(\mu_{A}(x)\right)$ represents the state of object in the second column, and the errors of different actions are given in the fourth column, and the losses of different actions are given in the fifth column.

Each of the four losses $m_{\theta}\left(\widetilde{\lambda}_{e}\right), m_{\theta}\left(\widetilde{\lambda}_{r}\right), m_{\theta}\left(\widetilde{\lambda}_{s_{\downarrow}}\right)$ and $m_{\theta}\left(\widetilde{\lambda}_{s_{\uparrow}}\right)$ provides the unit cost, and the actual cost of each action is weighted by the magnitude of its error. Suppose $R_{a}(x)=m_{\theta}\left(\tilde{\lambda}_{a}\right) E_{a}\left(\mu_{A}(x)\right)$ denote the loss for taking actions $\left\{e, r, s_{\downarrow}, s_{\uparrow}\right\}$, the losses of four actions for an object can be computed as

$$
\begin{aligned}
R_{e}(x) & =m_{\theta}\left(\widetilde{\lambda}_{e}\right) E_{e}\left(\mu_{A}(x)\right)=\left(1-m_{\theta}\left(\mu_{A}(x)\right)\right) m_{\theta}\left(\widetilde{\lambda}_{e}\right) ; \\
R_{r}(x) & =m_{\theta}\left(\widetilde{\lambda}_{r}\right) E_{r}\left(\mu_{A}(x)\right)=m_{\theta}\left(\mu_{A}(x)\right) m_{\theta}\left(\widetilde{\lambda}_{r}\right) ; \\
R_{s_{\downarrow}}(x) & =m_{\theta}\left(\widetilde{\lambda}_{s_{\downarrow}}\right) E_{s_{\downarrow}}\left(m_{\theta}\left(\mu_{A}(x)\right)\right)=\left(m_{\theta}\left(\mu_{A}(x)\right)-0.5\right) m_{\theta}\left(\widetilde{\lambda}_{s_{\downarrow}}\right) ; \\
R_{s_{\uparrow}}(x) & \left.=m_{\theta}\left(\widetilde{\lambda}_{s_{\uparrow}}\right) E_{s_{\uparrow}}\left(m_{\theta}\left(\mu_{A}(x)\right)\right)=\left(0.5-m_{\theta}\left(\mu_{A}(x)\right)\right) m_{\theta} \widetilde{\lambda}_{s_{\uparrow}}\right) .
\end{aligned}
$$

Since only an action is taken for each object, the total loss of the approximation is computed by

$$
R=\sum_{x \in U} R_{a}(x)=\sum_{x \in U} m_{\theta}\left(\widetilde{\lambda}_{a}\right) E_{a}\left(\mu_{A}(x)\right)
$$

To minimize the total loss $R$, we take an action $\tau(x)$ that minimizes the loss $R_{a}(x)$ for each object, and $\tau(x)$ is a solution to the following minimization problem as

$$
\arg \min _{a \in a c t i o n} R_{a}(x)
$$


where $a \in\left\{e, r, s_{\downarrow}, s_{\uparrow}\right\}$.

According to the value $\mu_{A}(x)$ of an object $x$, we have two groups of decision rules for obtaining threeway approximations of an interval-valued fuzzy set as follows:

(1) When $m_{\theta}\left(\mu_{A}(x)\right) \geq 0.5$, (E1) If $R\left(a_{e} \mid x\right) \leq R\left(a_{r} \mid x\right)$ and $R\left(a_{e} \mid x\right) \leq R\left(a_{s_{\downarrow}} \mid x\right)$, then take action $a_{e}$; (R1) If $R\left(a_{r} \mid x\right) \leq R\left(a_{e} \mid x\right)$ and $R\left(a_{r} \mid x\right) \leq R\left(a_{s_{\downarrow}} \mid x\right)$, then take action $a_{r} ;(S 1)$ If $R\left(a_{s_{\downarrow}} \mid x\right) \leq R\left(a_{e} \mid x\right)$ and $R\left(a_{s_{\downarrow}} \mid x\right) \leq R\left(a_{r} \mid x\right)$, then take action $a_{s_{\downarrow}}$.

(2) When $m_{\theta}\left(\mu_{A}(x)\right)<0.5$, (E2) If $R\left(a_{e} \mid x\right) \leq R\left(a_{r} \mid x\right)$ and $R\left(a_{e} \mid x\right) \leq R\left(a_{s_{\uparrow}} \mid x\right)$, then take action $a_{e}$; (R2) If $R\left(a_{r} \mid x\right) \leq R\left(a_{e} \mid x\right)$ and $R\left(a_{r} \mid x\right) \leq R\left(a_{s_{\uparrow}} \mid x\right)$, then take action $a_{r} ;(S 2)$ : If $R\left(a_{s_{\uparrow}} \mid x\right) \leq R\left(a_{e} \mid x\right)$ and $R\left(a_{s_{\uparrow}} \mid x\right) \leq R\left(a_{r} \mid x\right)$, then take action $a_{s_{\uparrow}}$.

\subsection{Loss functions-based three-way approximations of interval-valued fuzzy sets}

In this subsection, we consider interval-valued loss functions satisfying certain properties for obtaining an analytic solution defining a three-way approximation.

Suppose $(c 1): m_{\theta}\left(\widetilde{\lambda}_{e}\right)>0, m_{\theta}\left(\widetilde{\lambda}_{r}\right)>0, m_{\theta}\left(\widetilde{\lambda}_{s_{\downarrow}}\right)>0, m_{\theta}\left(\widetilde{\lambda}_{s \uparrow}\right)>0 ;(c 2): m_{\theta}\left(\widetilde{\lambda}_{s_{\downarrow}}\right) \leq m_{\theta}\left(\widetilde{\lambda}_{r}\right) ;(c 3):$ $m_{\theta}\left(\widetilde{\lambda}_{s_{\uparrow}}\right) \leq m_{\theta}\left(\widetilde{\lambda}_{e}\right)$, Condition $(c 1)$ requires that all costs are nonnegative; Condition (c2) illustrates that reducing a membership grade $\mu_{A}(x) \geq 0.5$ to 0.5 represents a smaller adjustment than reducing it to 0 , and a smaller cost is associated with action $a_{s_{\downarrow}}$; Condition (c3) illustrates that elevating a membership grade $\mu_{A}(x)<0.5$ to 0.5 represents a smaller adjustment than elevating it to 1 , and a smaller cost is associated with action $a_{s_{\uparrow}}$. With the assumptions $(c 1)-(c 3)$, we simplify the decision rules as follows:

(1) When $m_{\theta}\left(\mu_{A}(x)\right) \geq 0.5$, the rule $(E 1)$ is expressed as

$$
\begin{aligned}
R\left(a_{e} \mid x\right) \leq R\left(a_{r} \mid x\right) & \Leftrightarrow \quad\left(1-m_{\theta}\left(\mu_{A}(x)\right)\right) m_{\theta}\left(\widetilde{\lambda}_{e}\right) \leq\left(m_{\theta}\left(\mu_{A}(x)\right)-0\right) m_{\theta}\left(\widetilde{\lambda}_{r}\right) \\
& \Leftrightarrow \quad \mu_{A}(x) \geq \frac{m_{\theta}\left(\widetilde{\lambda}_{e}\right)}{\lambda_{e} m_{\theta}\left(\widetilde{\lambda}_{e}\right)+m_{\theta}\left(\widetilde{\lambda}_{r}\right)}=\gamma ; \\
R\left(a_{e} \mid x\right) \leq R\left(a_{s_{\downarrow}} \mid x\right) & \Leftrightarrow \quad\left(1-m_{\theta}\left(\mu_{A}(x)\right)\right) m_{\theta}\left(\widetilde{\lambda}_{e}\right) \leq\left(m_{\theta}\left(\mu_{A}(x)\right)-0.5\right) m_{\theta}\left(\widetilde{\lambda}_{s_{\downarrow}}\right) \\
& \Leftrightarrow \quad m_{\theta}\left(\mu_{A}(x)\right) \geq \frac{2 m_{\theta}\left(\widetilde{\lambda}_{e}\right)+m_{\theta}\left(\widetilde{\lambda}_{s_{\downarrow}}\right)}{2\left(m_{\theta}\left(\widetilde{\lambda}_{e}\right)+m_{\theta}\left(\widetilde{\lambda}_{s_{\downarrow}}\right)\right.}=\alpha .
\end{aligned}
$$

The rule $R(1)$ is expressed by

$$
\begin{aligned}
R\left(a_{r} \mid x\right) \leq R\left(a_{r} \mid x\right) & \Leftrightarrow m_{\theta}\left(\mu_{A}(x)\right) \leq \gamma \\
R\left(a_{r} \mid x\right) \leq R\left(a_{s_{\downarrow}} \mid x\right) & \Leftrightarrow \quad m_{\theta}\left(\mu_{A}(x)\right) \lambda_{r} \leq\left(m_{\theta}\left(\mu_{A}(x)\right)-0.5\right) m_{\theta}\left(\widetilde{\lambda}_{s_{\downarrow}}\right) \\
& \Leftrightarrow \quad m_{\theta}\left(\mu_{A}(x)\right) \leq \frac{-m_{\theta}\left(\widetilde{\lambda}_{s_{\downarrow}}\right)}{2\left(m_{\theta}\left(\widetilde{\lambda}_{r}\right)-m_{\theta}\left(\widetilde{\lambda}_{s_{\downarrow}}\right)(t)\right)}=\gamma^{-} .
\end{aligned}
$$

The rule $S(1)$ is expressed by

$$
R\left(a_{s_{\downarrow}} \mid x\right) \leq R\left(a_{e} \mid x\right) \Leftrightarrow m_{\theta}\left(\mu_{A}(x)\right) \leq \alpha ; R\left(a_{s_{\downarrow}} \mid x\right) \leq R\left(a_{s_{\downarrow}} \mid x\right) \Leftrightarrow m_{\theta}\left(\mu_{A}(x)\right) \geq \gamma^{-} .
$$


Since $\gamma^{-} \leq 0$ contradicts with the assumption $m_{\theta}\left(\mu_{A}(x)\right) \geq 0.5$, it is impossible to apply rule (R1) for reducing membership values. Therefore, when $m_{\theta}\left(\mu_{A}(x)\right) \geq 0.5$, the rules are simply expressed as (E1) If $m_{\theta}\left(\mu_{A}(x)\right) \geq \alpha$, then $T_{\mu_{A}}(x)=1$; (S1) If $0.5 \leq m_{\theta}\left(\mu_{A}(x)\right)<\alpha$, then $T_{\mu_{A}}(x)=0.5$.

(2) When $m_{\theta}\left(\mu_{A}(x)\right)<0.5$, the rule $(E 2)$ is expressed as

$$
\begin{aligned}
R\left(a_{e} \mid x\right) \leq R\left(a_{r} \mid x\right) & \left.\Leftrightarrow \quad\left(1-m_{\theta}\left(\mu_{A}(x)\right)\right) m_{\theta}\left(\widetilde{\lambda}_{e}\right) \leq\left(m_{\theta}\left(\mu_{A}(x)\right)-0\right) m_{\theta} \widetilde{\lambda}_{r}\right) \\
& \Leftrightarrow \quad m_{\theta}\left(\mu_{A}(x)\right) \geq \frac{m_{\theta}\left(\widetilde{\lambda}_{e}\right)}{m_{\theta}\left(\widetilde{\lambda}_{e}\right)+m_{\theta}\left(\widetilde{\lambda}_{r}\right)}=\gamma ; \\
R\left(a_{e} \mid x\right) \leq R\left(a_{s_{\uparrow}} \mid x\right) & \Leftrightarrow \quad\left(1-m_{\theta}\left(\mu_{A}(x)\right)\right) m_{\theta}\left(\widetilde{\lambda}_{e}\right) \leq\left(0.5-m_{\theta}\left(\mu_{A}(x)\right)\right) m_{\theta}\left(\widetilde{\lambda}_{s_{\uparrow}}\right) \\
& \Leftrightarrow \quad m_{\theta}\left(\mu_{A}(x)\right) \geq \frac{m_{\theta}\left(\widetilde{\lambda}_{e}\right)-0.5 m_{\theta}\left(\widetilde{\lambda}_{s_{\uparrow}}\right)}{m_{\theta}\left(\widetilde{\lambda}_{e}\right)-m_{\theta}\left(\widetilde{\lambda}_{s_{\uparrow}}\right)}=\gamma^{+} .
\end{aligned}
$$

The rule $R(2)$ is expressed as

$$
\begin{aligned}
R\left(a_{r} \mid x\right) \leq R\left(a_{e} \mid x\right) & \Leftrightarrow m_{\theta}\left(\mu_{A}(x)\right) \leq \gamma ; \\
R\left(a_{r} \mid x\right) \leq R\left(a_{s_{\uparrow}} \mid x\right) & \Leftrightarrow m_{\theta}\left(\mu_{A}(x)\right) m_{\theta}\left(\widetilde{\lambda}_{r}\right) \leq\left(0.5-m_{\theta}\left(\mu_{A}(x)\right)\right) m_{\theta}\left(\widetilde{\lambda}_{s_{\uparrow}}\right) \\
& \Leftrightarrow \quad m_{\theta}\left(\mu_{A}(x)\right) \leq \frac{m_{\theta}\left(\widetilde{\lambda}_{s_{\uparrow}}\right)}{2\left(m_{\theta}\left(\widetilde{\lambda}_{r}\right)+m_{\theta}\left(\widetilde{\lambda}_{s \uparrow}\right)\right)}=\beta .
\end{aligned}
$$

The rule $S(2)$ is expressed as

$$
R\left(a_{s_{\uparrow}} \mid x\right) \leq R\left(a_{e} \mid x\right) \Leftrightarrow m_{\theta}\left(\mu_{A}(x)\right) \leq \gamma^{+} ; R\left(a_{s_{\uparrow}} \mid x\right) \leq R\left(a_{s_{\uparrow}} \mid x\right) \Leftrightarrow m_{\theta}\left(\mu_{A}(x)\right) \geq \beta .
$$

Since $\gamma^{+} \geq 1$ contradicts with the assumption $m_{\theta}\left(\mu_{A}(x)\right)<0.5$, it is impossible to apply rule (E2) for elevating membership values. Therefore, when $m_{\theta}\left(\mu_{A}(x)\right)<0.5$, the remaining rules are simply expressed as (R2) If $m_{\theta}\left(\mu_{A}(x)\right) \leq \beta$, then $T_{\mu_{A}}(x)=0$; (S2) If $\beta \leq m_{\theta}\left(\mu_{A}(x)\right)<0.5$, then $T_{\mu_{A}}(x)=0.5$.

By combining the two sets of rules, we immediately have three rules as (E) If $m_{\theta}\left(\mu_{A}(x)\right) \geq \alpha(t)$, then $T_{\mu_{A}}(x)=1$; (R) If $m_{\theta}\left(\mu_{A}(x)\right) \leq \beta$, then $T_{\mu_{A}}(x)=0$; (S) If $\beta<m_{\theta}\left(\mu_{A}(x)\right)<\alpha$, then $T_{\mu_{A}}(x)=0.5$, where

$$
\alpha=\frac{2 m_{\theta}\left(\widetilde{\lambda}_{e}\right)+m_{\theta}\left(\widetilde{\lambda}_{s_{\downarrow}}\right)}{2\left(m_{\theta}\left(\widetilde{\lambda}_{e}\right)+m_{\theta}\left(\widetilde{\lambda}_{s_{\downarrow}}\right)\right)} \text { and } \beta=\frac{m_{\theta}\left(\widetilde{\lambda}_{s_{\uparrow}}\right)}{2\left(m_{\theta}\left(\widetilde{\lambda}_{r}\right)+m_{\theta}\left(\widetilde{\lambda}_{s_{\uparrow}}\right)\right)} .
$$

\section{Interval-valued loss functions-based three-way approximations of interval- valued fuzzy sets: II}

In this section, we introduce another framework for decision-theoretic rough sets-based three-way approximations of interval-valued fuzzy sets.

Definition 6.1 Let $\widetilde{\mu}_{1}=\left[\mu_{1}^{-}, \mu_{1}^{+}\right]$and $\widetilde{\mu}_{2}=\left[\lambda_{2}^{-}, \mu_{2}^{+}\right]$be interval-valued sets, then the degree of possibility 
of $\widetilde{\mu}_{1} \geq \widetilde{\mu}_{2}$ and $\widetilde{\mu}_{2} \geq \widetilde{\mu}_{1}$ are defined as

$$
\begin{aligned}
& p\left(\widetilde{\mu}_{1} \geq \widetilde{\mu}_{2}\right)=\max \left\{1-\max \left\{\frac{\mu_{2}^{+}-\mu_{1}^{-}}{\mu_{1}^{+}-\mu_{1}^{-}+\mu_{2}^{+}-\mu_{2}^{-}}, 0\right\}, 0\right\} ; \\
& p\left(\widetilde{\mu}_{2} \geq \widetilde{\mu}_{1}\right)=\max \left\{1-\max \left\{\frac{\mu_{1}^{+}-\mu_{2}^{-}}{\mu_{1}^{+}-\mu_{1}^{-}+\mu_{2}^{+}-\mu_{2}^{-}}, 0\right\}, 0\right\} .
\end{aligned}
$$

In the sense of Definition 6.1, we have

$$
p\left(\widetilde{\mu}_{1} \geq \widetilde{\mu}_{2}\right)=\left\{\begin{array}{cc}
0, & \frac{\mu_{2}^{+}-\mu_{1}^{-}}{\mu_{1}^{+}-\mu_{1}^{-}+\mu_{2}^{+}-\mu_{2}^{-}} \geq 1 ; \\
1-\frac{\mu_{2}^{+}-\mu_{1}^{-}}{\mu_{1}^{+}-\mu_{1}^{-}+\mu_{2}^{+}-\mu_{2}^{-}}, & 0<\frac{\mu_{2}^{+}-\mu_{1}^{-}}{\mu_{1}^{+}-\mu_{1}^{-}+\mu_{2}^{+}-\mu_{2}^{-}}<1 ; \\
1, & \frac{\mu_{2}^{+}-\mu_{1}^{-}}{\mu_{1}^{+}-\mu_{1}^{-}+\mu_{2}^{+}-\mu_{2}^{-}} \leq 0 .
\end{array}\right.
$$

Furthermore, we have the complementary matrix of the preference as

$$
P_{\widetilde{\mu}_{1} \tilde{\mu}_{2} \widetilde{\mu}_{3}}=\left[\begin{array}{lll}
p\left(\widetilde{\mu}_{1} \geq \widetilde{\mu}_{1}\right) & p\left(\widetilde{\mu}_{1} \geq \widetilde{\mu}_{2}\right) & p\left(\widetilde{\mu}_{1} \geq \widetilde{\mu}_{3}\right) \\
p\left(\widetilde{\mu}_{2} \geq \widetilde{\mu}_{1}\right) & p\left(\widetilde{\mu}_{2} \geq \widetilde{\mu}_{2}\right) & p\left(\widetilde{\mu}_{2} \geq \widetilde{\mu}_{3}\right) \\
p\left(\widetilde{\mu}_{3} \geq \widetilde{\mu}_{1}\right) & p\left(\widetilde{\mu}_{3} \geq \widetilde{\mu}_{2}\right) & p\left(\widetilde{\mu}_{3} \geq \widetilde{\mu}_{3}\right)
\end{array}\right] .
$$

Suppose $\widetilde{R}_{a}(x)=\widetilde{\lambda}_{a} E_{a}\left(\mu_{A}(x)\right)$ denote the loss for taking actions $\left\{e, r, s_{\downarrow}, s_{\uparrow}\right\}$, the losses of four actions for an object can be computed as

$$
\begin{aligned}
\widetilde{R}_{e}(x) & =\widetilde{\lambda}_{e} E_{e}\left(\mu_{A}(x)\right)=\left[\left(1-m_{\theta}\left(\mu_{A}(x)\right)\right) \lambda_{e}^{-},\left(1-m_{\theta}\left(\mu_{A}(x)\right)\right) \lambda_{e}^{+}\right] ; \\
\widetilde{R}_{r}(x) & =\widetilde{\lambda}_{r} E_{r}\left(\mu_{A}(x)\right)=\left[m_{\theta}\left(\mu_{A}(x)\right) \lambda_{r}^{-}, m_{\theta}\left(\mu_{A}(x)\right) \lambda_{r}^{+}\right] ; \\
\widetilde{R}_{s_{\downarrow}}(x) & =\widetilde{\lambda}_{s_{\downarrow}} E_{s_{\downarrow}}\left(m_{\theta}\left(\mu_{A}(x)\right)\right)=\left[\left(m_{\theta}\left(\mu_{A}(x)\right)-0.5\right) \lambda_{s_{\downarrow}}^{-},\left(m_{\theta}\left(\mu_{A}(x)\right)-0.5\right) \lambda_{s_{\downarrow}}^{+}\right] ; \\
\widetilde{R}_{s_{\uparrow}}(x) & =\widetilde{\lambda}_{s_{\uparrow}} E_{s_{\uparrow}}\left(m_{\theta}\left(\mu_{A}(x)\right)\right)=\left[\left(0.5-m_{\theta}\left(\mu_{A}(x)\right)\right) \lambda_{s_{\uparrow}}^{-},\left(0.5-m_{\theta}\left(\mu_{A}(x)\right)\right) \lambda_{s_{\uparrow}}^{+}\right] .
\end{aligned}
$$

Since only an action is taken for each object, the total loss of the approximation is computed by

$$
\widetilde{R}=\sum_{x \in U} \widetilde{R}_{a}(x)=\sum_{x \in U} \widetilde{\lambda}_{a} E_{a}\left(m_{\theta}\left(\mu_{A}(x)\right)\right) .
$$

To minimize the total loss $\widetilde{R}$, we take an action $\tau(x)$ that minimizes the loss $\widetilde{R}_{a}(x)$ for each object, and $\tau(x)$ is a solution to the following minimization problem as

$$
\arg \min _{a \in a c t i o n} \widetilde{R}_{a}(x)
$$

where $a \in\left\{e, r, s_{\downarrow}, s_{\uparrow}\right\}$.

According to the value $\mu_{A}(x)$ of an object $x$, we have two groups of decision rules for obtaining threeway approximations of an interval-valued fuzzy set.

In what follows, in light of complementary matrix of the preference, we discuss the ranking of the expected loss $\widetilde{R}_{a}(x)$ and generate decision rules in the context of interval-valued fuzzy sets. Concretely, there are two situations to discuss: $m_{\theta}\left(\mu_{A}(x)\right) \geq 0.5$ and $m_{\theta}\left(\mu_{A}(x)\right)<0.5$. 


\subsection{Situation 1: $m_{\theta}\left(\mu_{A}(x)\right) \geq 0.5$}

If $m_{\theta}\left(\mu_{A}(x)\right) \geq 0.5$, then we have the complementary matrix of the preference as

$$
P_{e r s_{\downarrow}}=\left[\begin{array}{ccc}
p_{e e} & p_{e r} & p_{e s_{\downarrow}} \\
p_{r e} & p_{r r} & p_{r \downarrow} \\
p_{s_{\downarrow}} e & p_{s_{\downarrow} r} & p_{s_{\downarrow} s_{\downarrow}}
\end{array}\right] .
$$

According to the properties of the degree of possibilities, we have $p_{e e}=p_{r r}=p_{s_{\downarrow} s_{\downarrow}}=0.5, p_{e r}+p_{r e}=$ $1, p_{e s_{\downarrow}}+p_{s_{\downarrow} e}=1$ and $p_{r s_{\downarrow}}+p_{s_{\downarrow} r}=1$. Then we simplify the complementary matrix as

$$
P_{e r s \downarrow}=\left[\begin{array}{ccc}
0.5 & p_{e r} & p_{e s \downarrow} \\
1-p_{e r} & 0.5 & p_{r s_{\downarrow}} \\
1-p_{e s_{\downarrow}} & 1-p_{r s_{\downarrow}} & 0.5
\end{array}\right] .
$$

In light of the complementary matrix $P_{\text {ers }}$, all elements in each line of the matrix are summarized as

$$
p_{e}=0.5+p_{e r}+p_{e s_{\downarrow}} ; p_{r}=0.5-p_{e r}+p_{r s_{\downarrow}} ; p_{s_{\downarrow}}=2.5-p_{e s_{\downarrow}}-p_{r s_{\downarrow}},
$$

where $p_{e}$ is the total degree of preference of $\widetilde{R}_{e}(x) ; p_{r}$ is the total degree of preference of $\widetilde{R}_{r}(x) ; p_{s_{\downarrow}}$ is the total degree of preference of $\widetilde{R}_{s_{\downarrow}}(x)$. The values of $p_{e}, p_{r}$ and $p_{s_{\downarrow}}$ depend on $p_{e r}, p_{e s_{\downarrow}}$ and $p_{r s_{\downarrow}}$. We immediately have three rules as (E) If $p_{e} \leq p_{r}$ and $p_{e} \leq p_{s_{\downarrow}}$, then $T_{\mu_{A}}(x)=1$; (S) If $p_{s_{\downarrow}} \leq p_{e}$ and $p_{s_{\downarrow}} \leq p_{r}$, then $T_{\mu_{A}}(x)=0.5 ;(\mathrm{R})$ If $p_{r} \leq p_{e}$ and $p_{r} \leq p_{s_{\downarrow}}$, then $T_{\mu_{A}}(x)=0$.

Table 4: The complementary matrix for situation 1.

\begin{tabular}{cccc}
\hline $\mathrm{p}$ & $\widetilde{R}_{e}(x)$ & $\widetilde{R}_{r}(x)$ & $\widetilde{R}_{s_{\downarrow}}(x)$ \\
\hline$\widetilde{R}_{e}(x)$ & $p_{e e}=p\left(\widetilde{R}_{e}(x) \geq \widetilde{R}_{e}(x)\right)$ & $p_{e r}=p\left(\widetilde{R}_{e}(x) \geq \widetilde{R}_{r}(x)\right)$ & $p_{e s_{\downarrow}}=p\left(\widetilde{R}_{e}(x) \geq \widetilde{R}_{s_{\downarrow}}(x)\right)$ \\
$\widetilde{R}_{r}(x)$ & $p_{r e}=p\left(\widetilde{R}_{r}(x) \geq \widetilde{R}_{e}(x)\right)$ & $p_{r r}=p\left(\widetilde{R}_{r}(x) \geq \widetilde{R}_{r}(x)\right)$ & $p_{r s_{\downarrow}}=p\left(\widetilde{R}_{r}(x) \geq \widetilde{R}_{s_{\downarrow}}(x)\right)$ \\
$\widetilde{R}_{s_{\downarrow}}(x)$ & $p_{s_{\downarrow} e}=p\left(\widetilde{R}_{s_{\downarrow}}(x) \geq \widetilde{R}_{e}(x)\right)$ & $p_{s_{\downarrow} r}=p\left(\widetilde{R}_{s_{\downarrow}}(x) \geq \widetilde{R}_{r}(x)\right)$ & $p_{s_{\downarrow} s_{\downarrow}}=p\left(\widetilde{R}_{s_{\downarrow}}(x) \geq \widetilde{R}_{s_{\downarrow}}(x)\right)$ \\
\hline
\end{tabular}

In consideration of Definition 6.1, $p_{e r}=p\left(\widetilde{R}_{e}(x) \geq \widetilde{R}_{r}(x)\right)$ has three kinds of possible results: (I): $p_{e r}=0$, (II): $0<p_{e r}<1$, and (III): $p_{e r}=1$. Furthermore, we have the similar results for $p_{e s_{\downarrow}}$ and $p_{r s_{\downarrow}}$.

(1) For $p_{e r}$, if $p_{e r}=0$, we have

$$
\frac{m_{\theta}\left(\mu_{A}(x)\right) \lambda_{r}^{+}-\left(1-m_{\theta}\left(\mu_{A}(x)\right)\right) \lambda_{e}^{-}}{\left(1-m_{\theta}\left(\mu_{A}(x)\right)\right) \lambda_{e}^{+}-\left(1-m_{\theta}\left(\mu_{A}(x)\right)\right) \lambda_{e}^{-}+m_{\theta}\left(\mu_{A}(x)\right) \lambda_{r}^{+}-m_{\theta}\left(\mu_{A}(x)\right) \lambda_{r}^{-}} \geq 1 .
$$

In other words, we have

$$
m_{\theta}\left(\mu_{A}(x)\right) \lambda_{r}^{-} \geq\left(1-m_{\theta}\left(\mu_{A}(x)\right)\right) \lambda_{e}^{+} \Leftrightarrow m_{\theta}\left(\mu_{A}(x)\right) \geq \frac{\lambda_{e}^{+}}{\lambda_{r}^{-}+\lambda_{e}^{+}} .
$$

If $1>p_{e r}>0$, we have

$$
1>\frac{m_{\theta}\left(\mu_{A}(x)\right) \lambda_{r}^{+}-\left(1-m_{\theta}\left(\mu_{A}(x)\right)\right) \lambda_{e}^{-}}{\left(1-m_{\theta}\left(\mu_{A}(x)\right)\right) \lambda_{e}^{+}-\left(1-m_{\theta}\left(\mu_{A}(x)\right)\right) \lambda_{e}^{-}+m_{\theta}\left(\mu_{A}(x)\right) \lambda_{r}^{+}-m_{\theta}\left(\mu_{A}(x)\right) \lambda_{r}^{-}}>0 .
$$


In other words, we have

$$
\begin{aligned}
\left(1-m_{\theta}\left(\mu_{A}(x)\right)\right) \lambda_{e}^{+} & >m_{\theta}\left(\mu_{A}(x)\right) \lambda_{r}^{-} \Leftrightarrow m_{\theta}\left(\mu_{A}(x)\right)<\frac{\lambda_{e}^{+}}{\lambda_{r}^{-}+\lambda_{e}^{+}} \\
m_{\theta}\left(\mu_{A}(x)\right) \lambda_{r}^{+} & >\left(1-m_{\theta}\left(\mu_{A}(x)\right)\right) \lambda_{e}^{-} \Leftrightarrow m_{\theta}\left(\mu_{A}(x)\right)<\frac{\lambda_{e}^{-}}{\lambda_{r}^{+}+\lambda_{e}^{-}} .
\end{aligned}
$$

If $p_{e r}=1$, we have

$$
\frac{m_{\theta}\left(\mu_{A}(x)\right) \lambda_{r}^{+}-\left(1-m_{\theta}\left(\mu_{A}(x)\right)\right) \lambda_{e}^{-}}{\left(1-m_{\theta}\left(\mu_{A}(x)\right)\right) \lambda_{e}^{+}-\left(1-m_{\theta}\left(\mu_{A}(x)\right)\right) \lambda_{e}^{-}+m_{\theta}\left(\mu_{A}(x)\right) \lambda_{r}^{+}-m_{\theta}\left(\mu_{A}(x)\right) \lambda_{r}^{-}} \leq 0 .
$$

In other words, we have

$$
\left(1-m_{\theta}\left(\mu_{A}(x)\right)\right) \lambda_{e}^{-} \geq m_{\theta}\left(\mu_{A}(x)\right) \lambda_{r}^{+} \Leftrightarrow m_{\theta}\left(\mu_{A}(x)\right) \geq \frac{\lambda_{e}^{-}}{\lambda_{r}^{+}+\lambda_{e}^{-}} .
$$

(2) For $p_{e s_{\downarrow}}$, if $p_{e s_{\downarrow}}=0$, we have

$$
\frac{\left(m_{\theta}\left(\mu_{A}(x)\right)-0.5\right) \lambda_{s_{\downarrow}}^{+}-\left(1-m_{\theta}\left(\mu_{A}(x)\right)\right) \lambda_{e}^{-}}{\left(1-m_{\theta}\left(\mu_{A}(x)\right)\right) \lambda_{e}^{+}-\left(1-m_{\theta}\left(\mu_{A}(x)\right)\right) \lambda_{e}^{-}+\left(m_{\theta}\left(\mu_{A}(x)\right)-0.5\right) \lambda_{s_{\downarrow}}^{+}-\left(m_{\theta}\left(\mu_{A}(x)\right)-0.5\right) \lambda_{s_{\downarrow}}^{-}} \geq 1 .
$$

In other words, we have

$$
\left(m_{\theta}\left(\mu_{A}(x)\right)-0.5\right) \lambda_{s_{\downarrow}}^{+} \geq\left(1-m_{\theta}\left(\mu_{A}(x)\right)\right) \lambda_{e}^{+} \Leftrightarrow m_{\theta}\left(\mu_{A}(x)\right) \geq \frac{0.5 \lambda_{s_{\downarrow}}^{-}+\lambda_{e}^{+}}{\lambda_{s_{\downarrow}}^{-}+\lambda_{e}^{+}} .
$$

If $1>p_{e s_{\downarrow}}>0$, we have

$$
1>\frac{\left(m_{\theta}\left(\mu_{A}(x)\right)-0.5\right) \lambda_{s_{\downarrow}}^{+}-\left(1-m_{\theta}\left(\mu_{A}(x)\right)\right) \lambda_{e}^{-}}{\left(1-m_{\theta}\left(\mu_{A}(x)\right)\right) \lambda_{e}^{+}-\left(1-m_{\theta}\left(\mu_{A}(x)\right)\right) \lambda_{e}^{-}+\left(m_{\theta}\left(\mu_{A}(x)\right)-0.5\right) \lambda_{s_{\downarrow}}^{+}-\left(m_{\theta}\left(\mu_{A}(x)\right)-0.5\right) \lambda_{s_{\downarrow}}^{-}}>0 .
$$

In other words, we have

$$
\begin{aligned}
\left(m_{\theta}\left(\mu_{A}(x)\right)-0.5\right) \lambda_{s_{\downarrow}}^{+} & >\left(1-m_{\theta}\left(\mu_{A}(x)\right)\right) \lambda_{e}^{-} \Leftrightarrow m_{\theta}\left(\mu_{A}(x)\right)>\frac{0.5 \lambda_{s_{\downarrow}}^{+}+\lambda_{e}^{-}}{\lambda_{e}^{-}+\lambda_{s_{\downarrow}}^{+}} ; \\
\left(1-m_{\theta}\left(\mu_{A}(x)\right)\right) \lambda_{e}^{+} & >\left(m_{\theta}\left(\mu_{A}(x)\right)-0.5\right) \lambda_{s_{\downarrow}}^{+} \Leftrightarrow m_{\theta}\left(\mu_{A}(x)\right)<\frac{0.5 \lambda_{s_{\downarrow}}^{-}+\lambda_{e}^{+}}{\lambda_{s_{\downarrow}}^{-}+\lambda_{e}^{+}} .
\end{aligned}
$$

If $p_{e s_{\downarrow}}=1$, we have

$$
\frac{\left(m_{\theta}\left(\mu_{A}(x)\right)-0.5\right) \lambda_{s_{\downarrow}}^{+}-\left(1-m_{\theta}\left(\mu_{A}(x)\right)\right) \lambda_{e}^{-}}{\left(1-m_{\theta}\left(\mu_{A}(x)\right)\right) \lambda_{e}^{+}-\left(1-m_{\theta}\left(\mu_{A}(x)\right)\right) \lambda_{e}^{-}+\left(m_{\theta}\left(\mu_{A}(x)\right)-0.5\right) \lambda_{s_{\downarrow}}^{+}-\left(m_{\theta}\left(\mu_{A}(x)\right)-0.5\right) \lambda_{s_{\downarrow}}^{-}} \leq 0 .
$$

In other words, we have

$$
\left(m_{\theta}\left(\mu_{A}(x)\right)-0.5\right) \lambda_{s_{\downarrow}}^{+} \leq\left(1-m_{\theta}\left(\mu_{A}(x)\right)\right) \lambda_{e}^{-} \Leftrightarrow m_{\theta}\left(\mu_{A}(x)\right) \leq \frac{0.5 \lambda_{s_{\downarrow}}^{+}+\lambda_{e}^{-}}{\lambda_{s_{\downarrow}}^{+}-\lambda_{r}^{+}} .
$$


(3) For $p_{r s_{\downarrow}}$, if $p_{r s_{\downarrow}}=0$, we have

$$
\frac{\left(m_{\theta}\left(\mu_{A}(x)\right)-0.5\right) \lambda_{s_{\downarrow}}^{+}-m_{\theta}\left(\mu_{A}(x)\right) \lambda_{r}^{-}}{m_{\theta}\left(\mu_{A}(x)\right) \lambda_{r}^{+}-m_{\theta}\left(\mu_{A}(x)\right) \lambda_{r}^{-}+\left(m_{\theta}\left(\mu_{A}(x)\right)-0.5\right) \lambda_{s_{\downarrow}}^{+}-\left(m_{\theta}\left(\mu_{A}(x)\right)-0.5\right) \lambda_{s_{\downarrow}}^{-}} \geq 1 .
$$

In other words, we have

$$
m_{\theta}\left(\mu_{A}(x)\right) \lambda_{r}^{+} \leq\left(m_{\theta}\left(\mu_{A}(x)\right)-0.5\right) \lambda_{s_{\downarrow}}^{-} \Leftrightarrow m_{\theta}\left(\mu_{A}(x)\right) \leq \frac{0.5 \lambda_{s_{\downarrow}}^{-}}{\lambda_{s_{\downarrow}}^{-}-\lambda_{r}^{+}} .
$$

If $0<p_{r s_{\downarrow}}<1$, we have

$$
1>\frac{\left(m_{\theta}\left(\mu_{A}(x)\right)-0.5\right) \lambda_{s_{\downarrow}}^{+}-m_{\theta}\left(\mu_{A}(x)\right) \lambda_{r}^{-}}{m_{\theta}\left(\mu_{A}(x)\right) \lambda_{r}^{+}-m_{\theta}\left(\mu_{A}(x)\right) \lambda_{r}^{-}+\left(m_{\theta}\left(\mu_{A}(x)\right)-0.5\right) \lambda_{s_{\downarrow}}^{+}-\left(m_{\theta}\left(\mu_{A}(x)\right)-0.5\right) \lambda_{s_{\downarrow}}^{-}}>0 .
$$

In other words, we have

$$
\begin{aligned}
\left(m_{\theta}\left(\mu_{A}(x)\right)-0.5\right) \lambda_{s_{\downarrow}}^{+} & >m_{\theta}\left(\mu_{A}(x)\right) \lambda_{r}^{-} \Leftrightarrow m_{\theta}\left(\mu_{A}(x)\right)<\frac{0.5 \lambda_{s_{\downarrow}}^{+}}{\lambda_{s_{\downarrow}}^{+}-\lambda_{r}^{-}} ; \\
m_{\theta}\left(\mu_{A}(x)\right) \lambda_{r}^{+} & >\left(m_{\theta}\left(\mu_{A}(x)\right)-0.5\right) \lambda_{s_{\downarrow}}^{-} \Leftrightarrow m_{\theta}\left(\mu_{A}(x)\right)>\frac{0.5 \lambda_{s_{\downarrow}}^{-}}{\lambda_{s_{\downarrow}}^{+}-\lambda_{r}^{+}} .
\end{aligned}
$$

If $p_{r s_{\downarrow}}=1$, we have

$$
\frac{\left(m_{\theta}\left(\mu_{A}(x)\right)-0.5\right) \lambda_{s_{\downarrow}}^{+}-m_{\theta}\left(\mu_{A}(x)\right) \lambda_{r}^{-}}{m_{\theta}\left(\mu_{A}(x)\right) \lambda_{e}^{+}-m_{\theta}\left(\mu_{A}(x)\right) \lambda_{e}^{-}+\left(m_{\theta}\left(\mu_{A}(x)\right)-0.5\right) \lambda_{s_{\downarrow}}^{+}-\left(m_{\theta}\left(\mu_{A}(x)\right)-0.5\right) \lambda_{s_{\downarrow}}^{-}} \leq 0 .
$$

In other words, we have

$$
m_{\theta}\left(\mu_{A}(x)\right) \lambda_{r}^{-} \geq\left(m_{\theta}\left(\mu_{A}(x)\right)-0.5\right) \lambda_{s_{\downarrow}}^{+} \Leftrightarrow m_{\theta}\left(\mu_{A}(x)\right) \geq \frac{0.5 \lambda_{s_{\downarrow}}^{+}}{\lambda_{s_{\downarrow}}^{+}-\lambda_{r}^{-}} ;
$$

Example 6.2 (Continuation of Example 3.3) Let $\tilde{\lambda}_{e}=[1,2], \widetilde{\lambda}_{r}=[5,6]$ and $\widetilde{\lambda}_{s_{\downarrow}}=[3,4]$ when $m_{\theta}\left(\mu_{A}(x)\right) \geq$ 0.5 , we have that

$$
\begin{aligned}
\widetilde{R}_{e}\left(x_{2}\right) & =\widetilde{\lambda}_{e} E_{e}\left(m_{\theta}\left(\mu_{A}\left(x_{2}\right)\right)\right)=\left[0.3 \lambda_{e}^{-}, 0.3 \lambda_{e}^{+}\right]=[0.3,0.6] ; \\
\widetilde{R}_{r}\left(x_{2}\right) & =\widetilde{\lambda}_{r} E_{r}\left(m_{\theta}\left(\mu_{A}\left(x_{2}\right)\right)\right)=\left[0.7 \lambda_{r}^{-}, 0.7 \lambda_{r}^{+}\right]=[3.5,4.2] ; \\
\widetilde{R}_{s_{\downarrow}}\left(x_{2}\right) & =\widetilde{\lambda}_{s_{\downarrow}} E_{s_{\downarrow}}\left(m_{\theta}\left(\mu_{A}\left(x_{2}\right)\right)\right)=\left[0.2 \lambda_{s_{\downarrow}}^{-}, 0.2 \lambda_{s_{\downarrow}}^{+}\right]=[0.6,0.8] .
\end{aligned}
$$

In light of the complementary matrix $P_{\text {ers }}$, we have

$$
P_{e r s_{\downarrow}}=\left[\begin{array}{ccc}
p_{e e} & p_{e r} & p_{e s \downarrow} \\
p_{r e} & p_{r r} & p_{r s_{\downarrow}} \\
p_{s_{\downarrow} e} & p_{s_{\downarrow} r} & p_{s_{\downarrow} s_{\downarrow}}
\end{array}\right]=\left[\begin{array}{ccc}
0.5 & 0 & 0 \\
1 & 0.5 & 1 \\
1 & 0 & 0.5
\end{array}\right] .
$$

Therefore, we have that elevating the membership grade of $x_{2}$ to 1 is the best choice. 
Table 5: Types of operations for situation 1

\begin{tabular}{|c|c|c|c|c|}
\hline & $p_{e r}$ & $p_{e s_{\downarrow}}$ & $p_{r s_{\downarrow}}$ & $T_{\mu_{A}}(x)$ \\
\hline 1 & $\mathrm{I}$ & I & I & 1 \\
\hline 2 & I & I & II & 1 \\
\hline 3 & I & I & III & 1 \\
\hline 4 & I & II & I & 1 \\
\hline 5 & I & II & II & 1 or 0.5 \\
\hline 6 & I & II & III & 1 or 0.5 \\
\hline 7 & I & III & I & 1 \\
\hline 8 & I & III & II & 0.5 \\
\hline 9 & I & III & III & 0.5 \\
\hline 10 & II & I & I & 1 or 0 \\
\hline 11 & II & I & II & 1 or 0 or 0.5 \\
\hline 12 & II & I & III & 1 \\
\hline 13 & II & II & I & 1 or 0 \\
\hline 14 & II & II & II & 1 or 0.5 or 0 \\
\hline 15 & II & II & III & 1 or 0.5 \\
\hline 16 & II & III & I & 0 \\
\hline 17 & II & III & II & 0.5 or 0 \\
\hline 18 & II & III & III & 0.5 \\
\hline 19 & III & I & I & 0 \\
\hline 20 & III & I & II & 0 \\
\hline 21 & III & I & III & 1 \\
\hline 22 & III & II & I & 0 \\
\hline 23 & III & II & II & 0.5 or 0 \\
\hline 24 & III & II & III & 0.5 \\
\hline 25 & III & III & I & 0 \\
\hline 26 & III & III & II & 0.5 or 0 \\
\hline 27 & III & III & III & 0.5 \\
\hline
\end{tabular}


Table 6: Special types of operations for situation 1.

\begin{tabular}{|c|c|c|}
\hline Type & Condition & $T_{\mu_{A}}(x)$ \\
\hline \multirow[t]{2}{*}{5} & $2 p_{e s_{\downarrow}}+p_{r s_{\downarrow}} \leq 2$ & 1 \\
\hline & $2 p_{e s_{\downarrow}}+p_{r s_{\downarrow}}>2$ & 0.5 \\
\hline \multirow[t]{2}{*}{6} & $p_{e s_{\downarrow}} \leq \frac{1}{2}$ & 0 \\
\hline & $p_{e s \downarrow}>\frac{1}{2}$ & 0.5 \\
\hline \multirow[t]{2}{*}{10} & $p_{e r} \leq \frac{1}{2}$ & 1 \\
\hline & $p_{e r}>\frac{1}{2}$ & 0 \\
\hline \multirow[t]{2}{*}{11} & $2 p_{e s_{\downarrow}}-p_{r s_{\downarrow}} \leq 1$ & 1 \\
\hline & $2 p_{e s_{\downarrow}}-p_{r s_{\downarrow}}>1$ & 0 \\
\hline \multirow[t]{2}{*}{13} & $2 p_{e r}+p_{r s_{\downarrow}} \leq 1 \wedge p_{e r}+2 p_{e s_{\downarrow}} \leq 2$ & 1 \\
\hline & $2 p_{e r}+p_{r s_{\downarrow}} \leq 1 \wedge-p_{e r}+2 p_{e s_{\downarrow}} \leq 1$ & 0 \\
\hline \multirow[t]{3}{*}{14} & $2 p_{e r}+p_{e s_{\downarrow}}-p_{r s_{\downarrow}} \leq 1 \wedge p_{e r}+2 p_{e s_{\downarrow}}+p_{r s_{\downarrow}} \leq 2$ & 1 \\
\hline & $1 \leq 2 p_{e r}+p_{e s_{\downarrow}}-p_{r s_{\downarrow}} \leq 1 \wedge p_{e s_{\downarrow}}-p_{e r}+2 p_{r s_{\downarrow}} \leq 1$ & 0 \\
\hline & $2 \leq p_{e r}+2 p_{e s_{\downarrow}}+p_{r s_{\downarrow}} \wedge 1 \leq p_{e s_{\downarrow}}-p_{e r}+2 p_{r s_{\downarrow}} \leq 1$ & 0 \\
\hline \multirow[t]{2}{*}{15} & $2 p_{e r}+p_{e s_{\downarrow}} \leq 2 \wedge p_{e r}+2 p_{r s_{\downarrow}} \leq 1$ & 1 \\
\hline & $1 \leq 2 p_{e r}+p_{e s_{\downarrow}} \wedge p_{e r}-p_{e s \downarrow} \leq 1$ & 0.5 \\
\hline \multirow[t]{2}{*}{17} & $2 p_{r s_{\downarrow}}-p_{e r} \leq 0$ & 0 \\
\hline & $2 p_{r s_{\downarrow}}-p_{e r}>0$ & 0.5 \\
\hline \multirow[t]{2}{*}{23} & $2 p_{r s_{\downarrow}}+p_{e s_{\downarrow}} \leq 2$ & 0 \\
\hline & $2 p_{r s_{\downarrow}}+p_{e r}>2$ & 0.5 \\
\hline \multirow[t]{2}{*}{26} & $p_{r{ }_{\downarrow}} \leq 0.5$ & 0 \\
\hline & $p_{r s_{\downarrow}}>0.5$ & 0.5 \\
\hline
\end{tabular}


6.2 Situation 2: $m_{\theta}\left(\mu_{A}(x)\right)<0.5$

For $m_{\theta}\left(\mu_{A}(x)\right) \leq 0.5$, we have

$$
P_{e r s_{\uparrow}}=\left[\begin{array}{ccc}
p_{e e} & p_{e r} & p_{e s_{\uparrow}} \\
p_{r e} & p_{r r} & p_{r s_{\uparrow}} \\
p_{s_{\uparrow} e} & p_{s_{\uparrow} r} & p_{s_{\uparrow} s_{\uparrow}}
\end{array}\right] .
$$

According to the properties of the degree of possibilities, we have $p_{e e}=p_{r r}=p_{s_{\uparrow} s_{\uparrow}}=0.5, p_{e r}+p_{r e}=$ $1, p_{e s \uparrow}+p_{s \uparrow e}=1$ and $p_{r s \uparrow}+p_{s \uparrow r}=1$. Then we simplify the matrix as

$$
P_{e r s_{\uparrow}}=\left[\begin{array}{ccc}
0.5 & p_{e r} & p_{e s \uparrow} \\
1-p_{e r} & 0.5 & p_{r s_{\uparrow}} \\
1-p_{e s_{\uparrow}} & 1-p_{r s \uparrow} & 0.5
\end{array}\right] .
$$

In light of the complementary matrix $P_{\text {ers }}$, all elements in each line of the matrix are summarized as

$$
p_{e}=0.5+p_{e r}+p_{e s_{\uparrow}} ; p_{r}=0.5-p_{e r}+p_{r s_{\uparrow}} ; p_{s_{\uparrow}}=2.5-p_{e s_{\uparrow}}-p_{r s_{\uparrow}},
$$

where $p_{e}$ is the total degree of preference of $\widetilde{R}_{e}(x) ; p_{r}$ is the total degree of preference of $\widetilde{R}_{r}(x) ; p_{s_{\uparrow}}$ is the total degree of preference of $\widetilde{R}_{s_{\uparrow}}(x)$. The values of $p_{e}, p_{r}$ and $p_{s_{\uparrow}}$ depend on $p_{e r}, p_{e s \uparrow}$ and $p_{r s \uparrow}$. We immediately have three rules as (E) If $p_{e} \leq p_{r}$ and $p_{e} \leq p_{s_{\uparrow}}$, then $T_{\mu_{A}}(x)=1$; (S) If $p_{s_{\uparrow}} \leq p_{e}$ and $p_{s_{\uparrow}} \leq p_{r}$, then $T_{\mu_{A}}(x)=0.5 ;(\mathrm{R})$ If $p_{r} \leq p_{e}$ and $p_{r} \leq p_{s_{v}}$, then $T_{\mu_{A}}(x)=0$.

Table 7: The complementary matrix for situation 2 .

\begin{tabular}{cccc}
\hline $\mathrm{p}$ & $\widetilde{R}_{e}(x)$ & $\widetilde{R}_{r}(x)$ & $\widetilde{R}_{s_{\uparrow}}(x)$ \\
\hline$\widetilde{R}_{e}(x)$ & $p_{e e}=p\left(\widetilde{R}_{e}(x) \geq \widetilde{R}_{e}(x)\right)$ & $p_{e r}=p\left(\widetilde{R}_{e}(x) \geq \widetilde{R}_{r}(x)\right)$ & $p_{e s_{\uparrow}}=p\left(\widetilde{R}_{e}(x) \geq \widetilde{R}_{s_{\uparrow}}(x)\right)$ \\
$\widetilde{R}_{r}(x)$ & $p_{r e}=p\left(\widetilde{R}_{r}(x) \geq \widetilde{R}_{e}(x)\right)$ & $p_{r r}=p\left(\widetilde{R}_{r}(x) \geq \widetilde{R}_{r}(x)\right)$ & $p_{r s_{\uparrow}}=p\left(\widetilde{R}_{r}(x) \geq \widetilde{R}_{s_{\uparrow}}(x)\right)$ \\
$\widetilde{R}_{s_{\uparrow}}(x)$ & $p_{s_{\uparrow} e}=p\left(\widetilde{R}_{s_{\uparrow}}(x) \geq \widetilde{R}_{e}(x)\right)$ & $p_{s_{\uparrow} r}=p\left(\widetilde{R}_{s_{\uparrow}}(x) \geq \widetilde{R}_{r}(x)\right)$ & $p_{s_{\uparrow} s_{\uparrow}}=p\left(\widetilde{R}_{s_{\uparrow}}(x) \geq \widetilde{R}_{s_{\uparrow}}(x)\right)$ \\
\hline
\end{tabular}

In consideration of Definition 6.1, $p_{e r}=p\left(\widetilde{R}_{e}(x) \geq \widetilde{R}_{r}(x)\right)$ have three kinds of possible results: (I): $p_{e r}=0$, (II): $0<p_{e r}<1$; (III): $p_{e r}=1$. Furthermore, we have the similar results for $p_{e s_{\downarrow}}$ and $p_{r s_{\downarrow}}$.

(1) For $p_{e r}$, if $p_{e r}=0$, we have

$$
\frac{m_{\theta}\left(\mu_{A}(x)\right) \lambda_{r}^{+}-\left(1-m_{\theta}\left(\mu_{A}(x)\right)\right) \lambda_{e}^{-}}{\left(1-m_{\theta}\left(\mu_{A}(x)\right)\right) \lambda_{e}^{+}-\left(1-m_{\theta}\left(\mu_{A}(x)\right)\right) \lambda_{e}^{-}+m_{\theta}\left(\mu_{A}(x)\right) \lambda_{r}^{+}-m_{\theta}\left(\mu_{A}(x)\right) \lambda_{r}^{-}} \geq 1 .
$$

In other words, we have

$$
m_{\theta}\left(\mu_{A}(x)\right) \lambda_{r}^{-} \geq\left(1-m_{\theta}\left(\mu_{A}(x)\right)\right) \lambda_{e}^{+} \Leftrightarrow m_{\theta}\left(\mu_{A}(x)\right) \geq \frac{\lambda_{e}^{+}}{\lambda_{r}^{-}+\lambda_{e}^{+}} .
$$

If $0<p_{\text {er }}<1$, we have

$$
1>\frac{m_{\theta}\left(\mu_{A}(x)\right) \lambda_{r}^{+}-\left(1-m_{\theta}\left(\mu_{A}(x)\right)\right) \lambda_{e}^{-}}{\left(1-m_{\theta}\left(\mu_{A}(x)\right)\right) \lambda_{e}^{+}-\left(1-m_{\theta}\left(\mu_{A}(x)\right)\right) \lambda_{e}^{-}+m_{\theta}\left(\mu_{A}(x)\right) \lambda_{r}^{+}-m_{\theta}\left(\mu_{A}(x)\right) \lambda_{r}^{-}}>0 .
$$


In other words, we have

$$
\begin{aligned}
& m_{\theta}\left(\mu_{A}(x)\right) \lambda_{r}^{-}>\left(1-m_{\theta}\left(\mu_{A}(x)\right)\right) \lambda_{e}^{+} \Leftrightarrow m_{\theta}\left(\mu_{A}(x)\right)>\frac{\lambda_{e}^{+}}{\lambda_{e}^{+}+\lambda_{r}^{-}} \\
& m_{\theta}\left(\mu_{A}(x)\right) \lambda_{r}^{+}>\left(1-m_{\theta}\left(\mu_{A}(x)\right)\right) \lambda_{e}^{-} \Leftrightarrow m_{\theta}\left(\mu_{A}(x)\right)>\frac{\lambda_{e}^{-}}{\lambda_{r}^{+}+\lambda_{e}^{-}} .
\end{aligned}
$$

If $p_{e r}=1$, we have

$$
\frac{m_{\theta}\left(\mu_{A}(x)\right) \lambda_{r}^{+}-\left(1-m_{\theta}\left(\mu_{A}(x)\right)\right) \lambda_{e}^{-}}{\left(1-m_{\theta}\left(\mu_{A}(x)\right)\right) \lambda_{e}^{+}-\left(1-m_{\theta}\left(\mu_{A}(x)\right)\right) \lambda_{e}^{-}+m_{\theta}\left(\mu_{A}(x)\right) \lambda_{r}^{+}-m_{\theta}\left(\mu_{A}(x)\right) \lambda_{r}^{-}} \leq 0 .
$$

In other words, we have

$$
m_{\theta}\left(\mu_{A}(x)\right) \lambda_{r}^{+} \leq\left(1-m_{\theta}\left(\mu_{A}(x)\right)\right) \lambda_{e}^{-} \Leftrightarrow m_{\theta}\left(\mu_{A}(x)\right) \leq \frac{\lambda_{e}^{-}}{\lambda_{r}^{+}+\lambda_{e}^{-}} .
$$

(2) For $p_{e s \uparrow}$, if $p_{e s_{\uparrow}}=0$, we have

$$
\frac{\left(0.5-m_{\theta}\left(\mu_{A}(x)\right)\right) \lambda_{s_{\uparrow}}^{+}-\left(1-m_{\theta}\left(\mu_{A}(x)\right)\right) \lambda_{e}^{-}}{\left(1-m_{\theta}\left(\mu_{A}(x)\right)\right) \lambda_{e}^{+}-\left(1-m_{\theta}\left(\mu_{A}(x)\right)\right) \lambda_{e}^{-}+\left(0.5-m_{\theta}\left(\mu_{A}(x)\right)\right) \lambda_{s \uparrow}^{+}-\left(0.5-m_{\theta}\left(\mu_{A}(x)\right)\right) \lambda_{s_{\uparrow}}^{-}} \geq 1 .
$$

In other words, we have

$$
\left(0.5-m_{\theta}\left(\mu_{A}(x)\right)\right) \lambda_{s_{\uparrow}}^{+} \geq\left(1-m_{\theta}\left(\mu_{A}(x)\right)\right) \lambda_{e}^{+} \Leftrightarrow m_{\theta}\left(\mu_{A}(x)\right) \geq \frac{0.5 \lambda_{s_{\uparrow}}^{-}-\lambda_{e}^{+}}{\lambda_{s_{\uparrow}}^{-}-\lambda_{e}^{+}} .
$$

If $1>p_{e s_{\uparrow}}>0$, we have

$$
1>\frac{\left(0.5-m_{\theta}\left(\mu_{A}(x)\right)\right) \lambda_{s_{\uparrow}}^{+}-\left(1-m_{\theta}\left(\mu_{A}(x)\right)\right) \lambda_{e}^{-}}{\left(1-m_{\theta}\left(\mu_{A}(x)\right)\right) \lambda_{e}^{+}-\left(1-m_{\theta}\left(\mu_{A}(x)\right)\right) \lambda_{e}^{-}+\left(0.5-m_{\theta}\left(\mu_{A}(x)\right)\right) \lambda_{s_{\uparrow}}^{+}-\left(0.5-m_{\theta}\left(\mu_{A}(x)\right)\right) \lambda_{s_{\uparrow}}^{-}}>0 .
$$

In other words, we have

$$
\begin{aligned}
\left(0.5-m_{\theta}\left(\mu_{A}(x)\right)\right) \lambda_{s_{\uparrow}}^{+} & >\left(1-m_{\theta}\left(\mu_{A}(x)\right)\right) \lambda_{e}^{-} \Leftrightarrow m_{\theta}\left(\mu_{A}(x)\right)>\frac{\lambda_{e}^{-}-0.5 \lambda_{s_{\uparrow}}^{+}}{\lambda_{e}^{-}-\lambda_{s_{\uparrow}}^{+}} \\
\left(1-m_{\theta}\left(\mu_{A}(x)\right)\right) \lambda_{e}^{+} & >\left(0.5-m_{\theta}\left(\mu_{A}(x)\right)\right) \lambda_{s_{\uparrow}}^{+} \Leftrightarrow m_{\theta}\left(\mu_{A}(x)\right)>\frac{0.5 \lambda_{s_{\uparrow}}^{-}-\lambda_{e}^{-}}{\lambda_{s_{\uparrow}}^{-}-\lambda_{e}^{+}} .
\end{aligned}
$$

If $p_{e s_{\uparrow}}=1$, we have

$$
\frac{\left(0.5-m_{\theta}\left(\mu_{A}(x)\right)\right) \lambda_{s_{\uparrow}}^{+}-\left(1-m_{\theta}\left(\mu_{A}(x)\right)\right) \lambda_{e}^{-}}{\left(1-m_{\theta}\left(\mu_{A}(x)\right)\right) \lambda_{e}^{+}-\left(1-m_{\theta}\left(\mu_{A}(x)\right)\right) \lambda_{e}^{-}+\left(0.5-m_{\theta}\left(\mu_{A}(x)\right)\right) \lambda_{s_{\uparrow}}^{+}-\left(0.5-m_{\theta}\left(\mu_{A}(x)\right)\right) \lambda_{s_{\uparrow}}^{-}} \leq 0 .
$$

In other words, we have

$$
\left(1-m_{\theta}\left(\mu_{A}(x)\right)\right) \lambda_{e}^{+} \geq\left(0.5-m_{\theta}\left(\mu_{A}(x)\right)\right) \lambda_{s_{\uparrow}}^{+} \Leftrightarrow m_{\theta}\left(\mu_{A}(x)\right) \leq \frac{0.5 \lambda_{s_{\uparrow}}^{+}-\lambda_{e}^{-}}{\lambda_{s_{\uparrow}}^{+}-\lambda_{e}^{-}} .
$$


(3) For $p_{r s_{\uparrow}}$, if $p_{r s_{\uparrow}}=0$, we have

$$
\frac{\left(0.5-m_{\theta}\left(\mu_{A}(x)\right)\right) \lambda_{s_{\uparrow}}^{+}-m_{\theta}\left(\mu_{A}(x)\right) \lambda_{r}^{-}}{m_{\theta}\left(\mu_{A}(x)\right) \lambda_{r}^{+}-m_{\theta}\left(\mu_{A}(x)\right) \lambda_{r}^{-}+\left(0.5-m_{\theta}\left(\mu_{A}(x)\right)\right) \lambda_{s \uparrow}^{+}-\left(0.5-m_{\theta}\left(\mu_{A}(x)\right)\right) \lambda_{s_{\uparrow}}^{-}} \geq 1 .
$$

In other words, we have

$$
m_{\theta}\left(\mu_{A}(x)\right) \lambda_{r}^{+} \leq\left(0.5-m_{\theta}\left(\mu_{A}(x)\right)\right) \lambda_{s_{\uparrow}}^{-} \Leftrightarrow m_{\theta}\left(\mu_{A}(x)\right) \leq \frac{0.5 \lambda_{s_{\uparrow}}^{-}}{\lambda_{s_{\uparrow}}^{-}+\lambda_{r}^{+}} .
$$

If $1>p_{r s_{\downarrow}}>0$, we have

$$
1>\frac{\left(0.5-m_{\theta}\left(\mu_{A}(x)\right)\right) \lambda_{s_{\uparrow}}^{+}-m_{\theta}\left(\mu_{A}(x)\right) \lambda_{r}^{-}}{m_{\theta}\left(\mu_{A}(x)\right) \lambda_{r}^{+}-m_{\theta}\left(\mu_{A}(x)\right) \lambda_{r}^{-}+\left(0.5-m_{\theta}\left(\mu_{A}(x)\right)\right) \lambda_{s_{\uparrow}}^{+}-\left(0.5-m_{\theta}\left(\mu_{A}(x)\right)\right) \lambda_{s_{\uparrow}}^{-}}>0 .
$$

In other words, we have

$$
\begin{aligned}
& \left(0.5-m_{\theta}\left(\mu_{A}(x)\right)\right) \lambda_{s_{\uparrow}}^{+}>m_{\theta}\left(\mu_{A}(x)\right) \lambda_{r}^{-} \Leftrightarrow m_{\theta}\left(\mu_{A}(x)\right)<\frac{0.5 \lambda_{s_{\uparrow}}^{+}}{\lambda_{s_{\uparrow}}^{+}+\lambda_{r}^{-}} ; \\
& \left(0.5-m_{\theta}\left(\mu_{A}(x)\right)\right) \lambda_{s_{\uparrow}}^{-}>m_{\theta}\left(\mu_{A}(x)\right) \lambda_{r}^{+} \Leftrightarrow m_{\theta}\left(\mu_{A}(x)\right)>\frac{0.5 \lambda_{s_{\uparrow}}^{-}}{\lambda_{s_{\uparrow}}^{-}+\lambda_{r}^{+}} .
\end{aligned}
$$

If $p_{r s_{\downarrow}}=1$, we have

$$
\frac{\left(0.5-m_{\theta}\left(\mu_{A}(x)\right)\right) \lambda_{s_{\uparrow}}^{+}-m_{\theta}\left(\mu_{A}(x)\right) \lambda_{r}^{-}}{\left(1-m_{\theta}\left(\mu_{A}(x)\right)\right) \lambda_{r}^{+}-\left(1-m_{\theta}\left(\mu_{A}(x)\right)\right) \lambda_{r}^{-}+\left(0.5-m_{\theta}\left(\mu_{A}(x)\right)\right) \lambda_{s_{\uparrow}}^{+}-\left(0.5-m_{\theta}\left(\mu_{A}(x)\right)\right) \lambda_{s_{\uparrow}}^{-}} \leq 0 .
$$

In other words, we have

$$
m_{\theta}\left(\mu_{A}(x)\right) \lambda_{r}^{-} \geq\left(0.5-m_{\theta}\left(\mu_{A}(x)\right)\right) \lambda_{s_{\uparrow}}^{+} \Leftrightarrow m_{\theta}\left(\mu_{A}(x)\right) \geq \frac{0.5 \lambda_{s_{\uparrow}}^{+}}{\lambda_{r}^{-}+\lambda_{s_{\uparrow}}^{+}} .
$$

Example 6.3 (Continuation of Example 3.3) Let $\widetilde{\lambda}_{e}=[5,6], \widetilde{\lambda}_{r}=[1,2]$ and $\widetilde{\lambda}_{s_{\uparrow}}=[3,4]$ when $m_{\theta}\left(\mu_{A}(x)\right)<$ 0.5 , we have

$$
\begin{aligned}
\widetilde{R}_{e}\left(x_{3}\right) & =\widetilde{\lambda}_{e} E_{e}\left(m_{\theta}\left(\mu_{A}\left(x_{3}\right)\right)\right)=\left[0.6 \lambda_{e}^{-}, 0.6 \lambda_{e}^{+}\right]=[3,3.6] ; \\
\widetilde{R}_{r}\left(x_{3}\right) & =\widetilde{\lambda}_{r} E_{r}\left(m_{\theta}\left(\mu_{A}\left(x_{3}\right)\right)\right)=\left[0.4 \lambda_{r}^{-}, 0.4 \lambda_{r}^{+}\right]=[0.4,0.8] ; \\
\widetilde{R}_{s_{\uparrow}}\left(x_{3}\right) & =\widetilde{\lambda}_{s_{\uparrow}} E_{s_{\uparrow}}\left(m_{\theta}\left(\mu_{A}\left(x_{3}\right)\right)\right)=\left[0.1 \lambda_{s_{\uparrow}}^{-}, 0.1 \lambda_{s_{\uparrow}}^{+}\right]=[0.3,0.4] .
\end{aligned}
$$

In light of the complementary matrix $P_{\text {ers } \uparrow}$, we have

$$
P_{e r s \uparrow}=\left[\begin{array}{ccc}
p_{e e} & p_{e r} & p_{e s \uparrow} \\
p_{r e} & p_{r r} & p_{r s_{\uparrow}} \\
p_{s_{\uparrow} e} & p_{s_{\uparrow} r} & p_{s_{\uparrow} s_{\uparrow}}
\end{array}\right]=\left[\begin{array}{ccc}
0.5 & 1 & 1 \\
0 & 0.5 & 1 \\
0 & 0 & 0.5
\end{array}\right] .
$$

Therefore, we have that elevating the membership of $x_{3}$ to 0.5 is the best choice. 
Table 8: Types of operations for situation 2.

\begin{tabular}{|c|c|c|c|c|}
\hline & $p_{e r}$ & $p_{e s \uparrow}$ & $p_{r s \uparrow}$ & $T_{\mu_{A}}(x)$ \\
\hline 1 & $\mathrm{I}$ & I & I & 1 \\
\hline 2 & I & I & II & 1 \\
\hline 3 & I & I & III & 1 \\
\hline 4 & I & II & I & 1 \\
\hline 5 & I & II & II & 1 or 0.5 \\
\hline 6 & I & II & III & 1 or 0.5 \\
\hline 7 & I & III & I & 1 \\
\hline 8 & I & III & II & 0.5 \\
\hline 9 & I & III & III & 0.5 \\
\hline 10 & II & I & I & 1 or 0 \\
\hline 11 & II & I & II & 1 or 0 or 0.5 \\
\hline 12 & II & I & III & 1 \\
\hline 13 & II & II & I & 1 or 0 \\
\hline 14 & II & II & II & 1 or 0.5 or 0 \\
\hline 15 & II & II & III & 1 or 0.5 \\
\hline 16 & II & III & I & 0 \\
\hline 17 & II & III & II & 0.5 or 0 \\
\hline 18 & II & III & III & 0.5 \\
\hline 19 & III & I & I & 0 \\
\hline 20 & III & I & II & 0 \\
\hline 21 & III & I & III & 1 \\
\hline 22 & III & II & I & 0 \\
\hline 23 & III & II & II & 0.5 or 0 \\
\hline 24 & III & II & III & 0.5 \\
\hline 25 & III & III & I & 0 \\
\hline 26 & III & III & II & 0.5 or 0 \\
\hline 27 & III & III & III & 0.5 \\
\hline
\end{tabular}


Table 9: Special types of operations for situation 2.

\begin{tabular}{|c|c|c|}
\hline Type & Condition & $T_{\mu_{A}}(x)$ \\
\hline \multirow[t]{2}{*}{5} & $2 p_{e s_{\uparrow}}+p_{r s_{\uparrow}} \leq 2$ & 1 \\
\hline & $2 p_{e s \uparrow}+p_{r s_{\uparrow}}>2$ & 0.5 \\
\hline \multirow[t]{2}{*}{6} & $p_{e s_{\uparrow}} \leq \frac{1}{2}$ & 0 \\
\hline & $p_{e s \uparrow}>\frac{1}{2}$ & 0.5 \\
\hline \multirow[t]{2}{*}{10} & $p_{e r} \leq \frac{1}{2}$ & 1 \\
\hline & $p_{e r}>\frac{1}{2}$ & 0 \\
\hline \multirow[t]{2}{*}{11} & $2 p_{e s_{\uparrow}}-p_{r s_{\uparrow}} \leq 1$ & 1 \\
\hline & $2 p_{e s_{\uparrow}}-p_{r s_{\uparrow}}>1$ & 0 \\
\hline \multirow[t]{2}{*}{13} & $2 p_{e r}+p_{r s_{\uparrow}} \leq 1 \wedge p_{e r}+2 p_{e s_{\uparrow}} \leq 2$ & 1 \\
\hline & $2 p_{e r}+p_{r s_{\uparrow}} \leq 1 \wedge-p_{e r}+2 p_{e s_{\uparrow}} \leq 1$ & 0 \\
\hline \multirow[t]{3}{*}{14} & $2 p_{e r}+p_{e s \uparrow}-p_{r s \uparrow} \leq 1 \wedge p_{e r}+2 p_{e s \uparrow}+p_{r s \uparrow} \leq 2$ & 1 \\
\hline & $1 \leq 2 p_{e r}+p_{e s_{\uparrow}}-p_{r s_{\uparrow}} \leq 1 \wedge p_{e s_{\uparrow}}-p_{e r}+2 p_{r s_{\downarrow}} \leq 1$ & 0 \\
\hline & $2 \leq p_{e r}+2 p_{e s_{\downarrow}}+p_{r s_{\downarrow}} \wedge 1 \leq p_{e s_{\uparrow}}-p_{e r}+2 p_{r s_{\uparrow}} \leq 1$ & 0 \\
\hline \multirow[t]{2}{*}{15} & $2 p_{e r}+p_{e s_{\uparrow}} \leq 2 \wedge p_{e r}+2 p_{r s_{\uparrow}} \leq 1$ & 1 \\
\hline & $1 \leq 2 p_{e r}+p_{e s_{\uparrow}} \wedge p_{e r}-p_{e s_{\uparrow}} \leq 1$ & 0.5 \\
\hline \multirow[t]{2}{*}{17} & $2 p_{r_{\uparrow}}-p_{e r} \leq 0$ & 0 \\
\hline & $2 p_{r s_{\uparrow}}-p_{e r}>0$ & 0.5 \\
\hline \multirow[t]{2}{*}{23} & $2 p_{r s_{\uparrow}}+p_{e s_{\uparrow}} \leq 2$ & 0 \\
\hline & $2 p_{r s_{\uparrow}}+p_{e r}>2$ & 0.5 \\
\hline \multirow[t]{2}{*}{26} & $p_{r s \uparrow} \leq 0.5$ & 0 \\
\hline & $p_{r s_{\uparrow}}>0.5$ & 0.5 \\
\hline
\end{tabular}




\section{Four semantics issues of this model}

In this section, we investigate four semantics issues of decision-theoretic three-way approximations of interval-valued fuzzy sets.

(1) Interpretations of interval-valued loss functions

In decision-theoretic three-way approximations of interval-valued fuzzy sets, the pair of thresholds depends on the choice of interval-valued loss functions which are fundamental notions of the decisiontheoretic model. In other words, given an interval-valued loss function, the pair of thresholds can be computed accordingly. On the other hand, if the pair of thresholds is interpreted in terms of an intervalvalued loss function, then the user can provide a better estimation of the thresholds in time. Therefore, the decision-theoretic model gives an interpretation of the pair of thresholds, and it is important to discuss approximations of interval-valued fuzzy sets by using interval-valued loss functions.

(2) Relationships to shadowed sets of fuzzy sets

In Sections 3 and 4, we see that three regions of decision-theoretic rough set-based three-way approximation $T_{\mu_{(A)}}$ and shadowed set $S_{\mu_{(A)}}$ are both defined through a pair of thresholds $\alpha$ and $\beta$. For shadowed sets, the objective function is given with respect to the membership functions, and different membership functions will produce different shadowed sets. In contrast, the objective function of the decision-theoretic framework is given with respect to interval-valued loss functions, which is independent of any particular fuzzy membership functions.

(3) Relationships to decision-theoretic rough sets

In Section 4, we adopt the main ideas from decision-theoretic rough set in developing decisiontheoretic rough set-based three-way approximations of interval-valued fuzzy sets. A rough membership function can be viewed as a fuzzy membership function. There are some differences between three-way approximations of interval-valued fuzzy sets and decision-theoretic rough sets. For decision-theoretic rough sets, we deal with two-state three-way decision problems. A rough membership function denotes the probability that an object is in the set. On the other hand, three-way approximations of interval-valued fuzzy sets are a many-state decision problem.

(4) Relationships to decision-theoretic three-way approximations of fuzzy sets

In [7], Deng et al. discussed three-way approximations of fuzzy sets by using loss functions. In practice, there are a lot of interval-valued loss functions. Compare with Deng's model, we discuss threeway approximations of interval-valued fuzzy sets by using interval-valued loss functions.

\section{Conclusions}

Many researchers have investigated approximations of interval-valued fuzzy sets. In this paper, firstly, we have presented shadowed sets for interpreting and understanding interval-valued fuzzy sets. Sec- 
ondly, we have constructed decision-theoretic rough set-based three-way approximations of intervalvalued fuzzy sets. Thirdly, we have computed the pair of thresholds for decision-theoretic rough set-based three-way approximations of interval-valued fuzzy sets by using interval-valued loss functions. Fourthly, we have constructed approximations of interval-valued fuzzy sets by using interval-valued loss functions from another view. Finally, we have employed several examples to illustrate that how to make a decision for interval-valued fuzzy sets by using interval-valued loss functions.

There are still many interesting topics deserving further investigations on fuzzy sets. For example, there are many types of fuzzy sets and loss functions, and it is of interest to investigate loss functionsbased three-way approximations of interval-valued fuzzy sets. In the future, we will further investigate interval-valued fuzzy sets and discuss its application in knowledge discovery.

\section{Acknowledgments}

We would like to thank the anonymous reviewers very much for their professional comments and valuable suggestions. This work is supported by the National Natural Science Foundation of China (NO. 11371130, 11401052, 11401195), the Scientific Research Fund of Hunan Provincial Education Department (No.14C0049).

\section{References}

[1] M. Banerjee, S.K. Pal, Roughness of a fuzzy set, Information Sciences 93 (1996) 235-246.

[2] G. Cattaneo, D. Ciucci, Shadowed sets and related algebraic structures, Fundamenta Informaticae 55 (2003) 255-284.

[3] G. Cattaneo, D. Ciucci, An algebraic approach to shadowed sets, Electronic Notes in Theoretical Computer Science 82 (2003) 64-75.

[4] G. Cattaneo, D. Ciucci, Theoretical aspects of shadowed sets, W. Pedrycz, A. Skowron, V. Kreinovich (Eds.), Handbook of Granular Computing, John Wiley and Sons, New York (2008), pp. 603-628.

[5] K. Chakrabarty, R. Biswas, S. Nanda, Nearest ordinary set of a fuzzy set: a rough theoretic construction, Bulletin of the Polish Academy of Sciences: Technical Sciences 46 (1998) 105-114.

[6] S. Chanas, On the interval approximation of a fuzzy number, Fuzzy Sets and Systems 122 (2001) 353-356. 
[7] X.F. Deng, Y.Y. Yao, Decision-theoretic three-way approximations of fuzzy sets, Information Sciences 279 (2014) 702-715.

[8] X.F. Deng, Y.Y. Yao, Mean-value-based decision-theoretic shadowed sets, W. Pedrycz, M.Z. Reformat (Eds.), Proceedings of 2013 Joint IFSA World Congress and NAFIPS Annual Meeting (IFSA/NAFIPS), IEEE Press, New York (2013) 1382-1387.

[9] D. Dubois, H. Prade, Fuzzy Sets and Fuzzy Rough Sets: Theory and Applications, Academic Press, New York (1980).

[10] P. Grzegorzewski, Nearest interval approximation of a fuzzy number, Fuzzy Sets and Systems 130 (2002) 321-330.

[11] P. Grzegorzewski, Fuzzy number approximation via shadowed sets, Information Sciences 225 (2013) $35-46$.

[12] G.J. Klir, Y. Bo, Fuzzy Sets and Fuzzy Logic: Theory and Applications, Prentice Hall, New Jersey (1995).

[13] H.X. Li, X.Z. Zhou, Risk decision making based on decision-theoretic rough set: a three-way view decision model, International Journal of Computational Intelligence Systems 4 (2011) 1-11.

[14] D.C. Liang, D. Liu, W. Pedrycz, P. Hu, Triangular fuzzy decision-theoretic rough sets, International Journal of Approximate Reasoning 54 (2013) 1087-1106.

[15] D.C. Liang, D. Liu, Systematic studies on three-way decisions with interval-valued decisiontheoretic rough sets, Information Sciences 276 (2014) 186-203.

[16] D. Liu, T.R. Li, D.C. Liang, Interval-valued decision-theoretic rough sets, Computer Science 39(7) (2012) 178-181.

[17] D. Liu, Y.Y. Yao, T.R. Li, Three-way investment decisions with decision-theoretic rough sets, International Journal of Computational Intelligence Systems 4 (2011) 66-74.

[18] D. Liu, T.R. Li, D.C. Liang, Three-Way Decisions in Dynamic Decision-Theoretic Rough Sets, Lecture Notes in Computer Science 8171 (2013) 291-301.

[19] S. Mitra, P.P. Kundu, Satellite image segmentation with shadowed C-means, Information Sciences 181 (2011) 3601-3613. 
[20] R. Moore, W. Lodwick, Interval analysis and fuzzy set theory, Fuzzy Sets and Systems 135 (2003) $5 \mathrm{C} 9$.

[21] E.N. Nasibov, S. Peker, On the nearest parametric approximation of a fuzzy number, Information Sciences 159 (2008) 1365-1375.

[22] W. Pedrycz, Shadowed sets: representing and processing fuzzy sets, IEEE Transactions on Systems Man Cybernetics-Systems 28 (1998) 103-109.

[23] W. Pedrycz, Shadowed sets: bridging fuzzy and rough sets, S.K. Pal, A. Skowron (Eds.), Rough Fuzzy Hybridization: A New Trend in Decision-Making, Springer, Singapore (1999) 179-199.

[24] W. Pedrycz, Fuzzy clustering with a knowledge-based guidance, Pattern Recognition Letters 25 (2004) 469-480.

[25] W. Pedrycz, Interpretation of clusters in the framework of shadowed sets, Pattern Recognition Letters 26 (2005) 2439-2449.

[26] W. Pedrycz, From fuzzy sets to shadowed sets: Interpretation and computing, International Journal of Intelligent Systems 24 (2009) 48-61.

[27] A. Pedrycz, F. Dong, K. Hirota, Finite cut-based approximation of fuzzy sets and its evolutionary optimization, Fuzzy Sets and Systems 160 (2009) 3550-3564.

[28] Y.H. Qian, J.Y. Liang, C.Y. Dang, Interval ordered information systems, Computers and Mathematics with Applications 56 (2008) 1994-2009.

[29] A. Sengupta, T.K. Pal, On comparing interval numbers, European Journal of Operational Research 127 (2000) 28-43.

[30] D. Slezak, W. Ziarko, The investigation of the Bayesian rough set model, International Journal of Approximate Reasoning 40 (2005) 81-91.

[31] I.B. Turksen, Interval valued fuzzy sets based on normal forms, Fuzzy Sets and Systems, 20(2) (1986) 191-210.

[32] L. Wang, J. Wang, Feature weighting fuzzy clustering integrating rough sets and shadowed sets, International Journal of Pattern Recognition and Artificial Intelligence 26 (2012) http://dx.doi.org/10.1142/S0218001412500103. 
[33] Y.Y. Yao, Probabilistic rough set approximations, International Journal of Approximate Reasoning 49 (2008) 255-271.

[34] Y.Y. Yao, Three-way decisions with probabilistic rough sets, Information Sciences 180 (2010) 341353.

[35] Y.Y. Yao, Two semantic issues in a probabilistic rough set model, Fundamenta Informaticae 108 (2011) 249-265.

[36] Y.Y. Yao, Probabilistic approaches to rough sets, Expert Systems 20 (2003) 287-297.

[37] Y.Y. Yao, The superiority of three-way decision in probabilistic rough set models, Information Sciences 181 (6) (2011) 1080-1096.

[38] W. Ziarko, Probabilistic approach to rough set, International Journal of Approximate Reasoning 49 (2008) 272-284.

[39] L.A. Zadeh, Fuzzy sets, Information and Control 8 (1965) 338-353.

[40] L.A. Zadeh, Is there a need for fuzzy logic? Information Sciences 178 (2008) 2751-2779.

[41] J. Zhou, W. Pedryczb, D.Q. Miao, Shadowed sets in the characterization of rough-fuzzy clustering, Pattern Recognition 44 (2011) 1738-1749. 